\title{
Physical characteristics of a dark cloud in an early stage of star formation toward NGC 7538
}

\section{An outer Galaxy infrared dark cloud?}

\author{
W. W. F. Frieswijk ${ }^{1,2}$, M. Spaans ${ }^{1}$, R. F. Shipman ${ }^{2}$, D. Teyssier ${ }^{2,3}$, and P. Hily-Blant ${ }^{4}$ \\ ${ }^{1}$ Kapteyn Astronomical Institute, University of Groningen, PO Box 800, 9700 AV Groningen, The Netherlands \\ e-mail: frieswyk@astro.rug.nl \\ 2 SRON, National Institute for Space Research, PO Box 800, 9700 AV Groningen, The Netherlands \\ 3 ESAC, European Space Astronomy Centre, Urb. Villafrancadel Castillo, PO Box 50727, Madrid 28080, Spain \\ ${ }^{4}$ IRAM, Institut de Radio Astronomie Millimétrique, 300 rue de la Piscine, St-Martin d’Hères, France
}

Received 22 January 2007 / Accepted 22 August 2007

\section{ABSTRACT}

\begin{abstract}
Context. In the inner parts of the Galaxy the Infrared Dark Clouds (IRDCs) are presently believed to be the progenitors of massive stars and star clusters. Many of them are predominantly devoid of active star formation and for now they represent the earliest observed stages of massive star formation. Their Outer Galaxy counterparts, if present, are not easily identified because of a low or absent mid-IR background.

Aims. We characterize the ambient conditions by determining physical parameters in the Outer Galaxy IRDC candidate G111.80+0.58, a relatively quiescent molecular core complex in the vicinity of active star forming regions such as NGC 7538 and S159.

Methods. We conduct molecular line observations on a number of dense cores in G111.80+0.58. We analyze the data in terms of excitation temperature, column and volume density, mass and stability.

Results. The temperatures we find (15-20 K) are higher than expected from only cosmic ray heating, but are comparable to those found in massive cores, such as IRDCs. Star forming activity could be present in some cores, as indicated by the presence of warm gas $\left(\mathrm{NH}_{3},{ }^{13} \mathrm{CO}\right.$ self-absorption) and Young Stellar Object candidates. The observed super-thermal line-widths are typical for star forming regions. The velocity dispersion is consistent with a turbulent energy cascade over the observed size scales of the complex. We do not find a correlation between the gas temperature and the line-width. The LTE masses we derive are much larger than the thermal Jeans mass. Therefore, fragmentation is expected and may have occurred already, in which case the observed lines represent the combined emission of multiple unresolved components.

Conclusions. We conclude that G111.80+0.58 is a molecular core complex with bulk properties very similar to IRDCs in an early, but not pristine, star forming state. The individual cores are close to virial equilibrium and some contain sufficient material to form massive stars and star clusters. The ambient conditions suggest that turbulence is involved in supporting the cores against gravitational collapse, at least down to the observed sizes. Additional high resolution data are necessary to resolve and analyze the smaller scale properties.
\end{abstract}

Key words. stars: formation - ISM: clouds - ISM: molecules - radio lines: ISM

\section{Introduction}

Infrared Dark Clouds (IRDCs) were discovered a decade ago as dark silhouettes against a bright mid-infrared background by the Midcourse Space Experiment (MSX, Egan et al. 1998) and the Infrared Space Observatory (ISO, Pérault et al. 1996). Many IRDCs contain compact (sub-) millimeter cores (Carey et al. 2000; Garay et al. 2004; Ormel et al. 2005; Rathborne et al. 2005) and the current picture is that these massive dense cores represent the early, cold stages of clustered star formation (Rathborne et al. 2006; Menten et al. 2005, and references therein) and that they are potentially the birth sites of massive

* Based on observations obtained with the 100-m telescope of MPIfR (Max-Planck-Institut für Radioastronomie) at Effelsberg and the IRAM $30 \mathrm{~m}$ telescope. IRAM is supported by INSU/CNRS (France), MPG (Germany) and IGN (Spain).

$\star \star$ Appendices are only available in electronic form at http://www. aanda.org stars. In order to assess the role of IRDCs in the process of star formation, their ambient physical conditions need to be determined. Only then insight may be gained into the differences between low- and high-mass star formation, the nature of the initial mass function and the impact of environment on star (cluster) formation. That is, the putative early stage that IRDCs represent implies that their physical state provides a direct record of the initial conditions pertinent to stellar birth.

A complete picture of the star forming properties in massive dark clouds, specifically the effects of external conditions on the formation process, requires a study of similar objects in different environments. The Outer Galaxy obviously represents the place in our Galaxy where the conditions, such as metallicity, density, interstellar radiation field and overall star formating activity are different compared to the inner regions of the Galaxy (e.g., Brand \& Wouterloot 1995; Rudolph et al. 2006). Variations of these properties from cloud to cloud may affect the local star formation rate, the mass distribution (IMF) or the star forming 
efficiency. In fact, only a direct comparison between Inner and Outer Galaxy star forming regions can asses in what way star formation differs with Galactic radius, if at all. However, due to a lack of bright background emission elsewhere, IRDCs were identified only in the inner parts of the Galaxy, mainly toward the Molecular Ring and the inner spiral arms (Simon et al. 2006). Can we identify massive dark clouds in an early evolutionary stage in the Outer Galaxy using a different approach?

Frieswijk \& Shipman (2007, in prep., FS07 hereafter) propose a list of candidate IRDC-like objects in the Outer Galactic Plane in their investigation of the distribution of highly reddened point sources observed in the Two Micron All Sky Survey (2MASS, Skrutskie et al. 2006). They suggest that a number of these clustered red sources are reddened due to foreground extinction in the form of dark clouds. As a verification of the presence of molecular material, they identified CO structures, observed in the Five College Radio Astronomy Observatory CO Survey of the Outer Galaxy (FCRAO, Heyer et al. 1998), that match the global morphology of the regions. Even though $\mathrm{CO}$ traces mainly the outer layers of molecular clouds, the amount of extinction they find indicates the presence of large column densities. A subset of the structures in this near-IR study are not associated with MSX or IRAS point sources and this would also suggest an early star forming nature. Additional data, e.g., of molecular lines and dust continuum are required to confirm if some of these near-IR structures are indeed the Outer Galaxy counterparts to the Inner Galaxy IRDCs.

In this paper we present follow-up molecular line observations of the Outer Galaxy dark cloud candidate G111.80+0.58 in the direction of Cepheus in the Perseus spiral arm. We derive physical properties for a number of dense cores located along the filamentary cloud structure, such as temperature, density and mass and we characterize the nature of the cloud by comparing the results to existing studies. The cloud is part of the Cas OB2 complex at a radial velocity around $-55 \mathrm{~km} \mathrm{~s}^{-1}$ (e.g., Blitz et al. 1982). There are a few well-known star forming regions located nearby, such as NGC 7538 and S159. NGC 7538 is actively forming stars and extensive studies have been conducted and reveal various stages of evolution including compact dense cores, HII regions, several massive proto-stellar objects and many lower mass Young Stellar Objects (YSO's) (e.g., Werner et al. 1979; Kameya et al. 1990; Sandell \& Sievers 2004). S159 is a bright reflection nebulae illuminated by an optically visible O-type star (Chini \& Wink 1984). Part of the cloud is associated with IRAS $23133+6050$ and seen as an emission nebula. In the immediate surrounding of S159 several compact radio continuum sources and (compact) HII regions have been identified (e.g., Lebrón et al. 2001).

Section 2 describes how the object was identified and gives a brief overview of the the appearance of the region and its immediate environment in MSX, 2MASS and FCRAO data. The observational setup and the target positions are given in Sect. 3 and Sect. 4 describes the observational properties. The derived physical properties of the cores are given in Sect. 5. In Sect. 6 we discuss the results and present our conclusion on the nature of dark cloud G111.80+0.58. Section 7 ends with some concluding remarks.

\section{G111.80+0.58: source selection}

G111.80+0.58 was selected from a list of candidate dark clouds. This list comprises all sources that were identified in the 2MASS Point Source Catalog (PSC) as extended red features in the Outer Galactic Plane (FS07). In this work, a statistical measure,

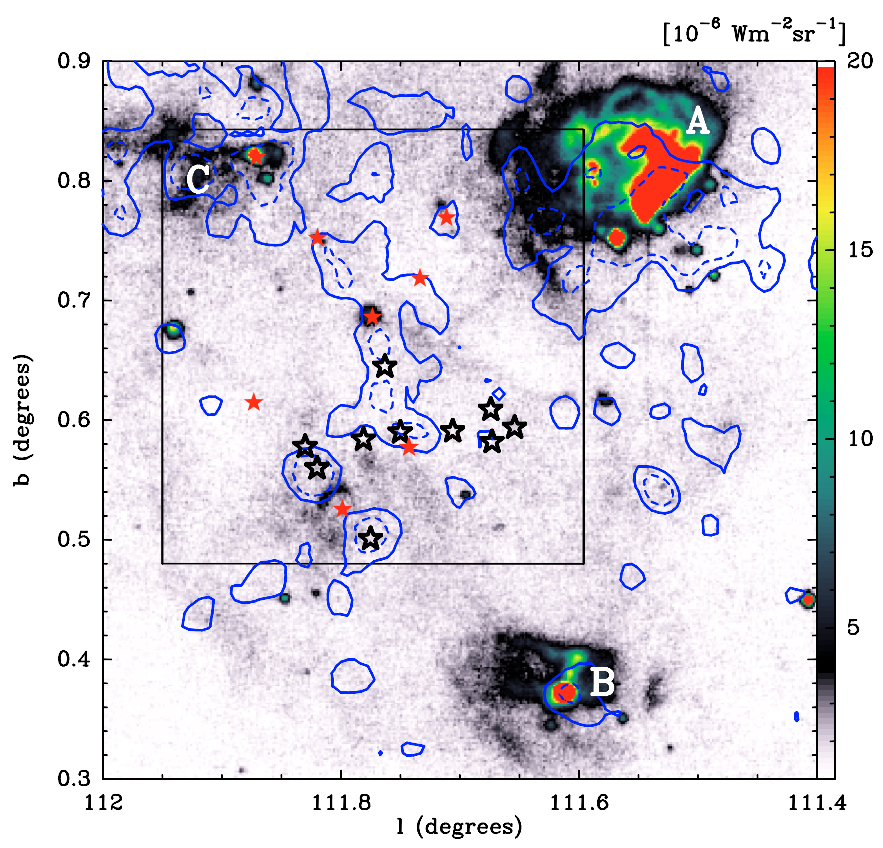

Fig. 1. $8 \mu \mathrm{m}$ emission of the region toward G111.80+0.58. The two prominent sources labeled A and B are NGC 7538 and S159, respectively. Region $\mathrm{C}$ is associated with several masers and radio sources. The region of interest for the current work is outlined by the box and is mostly devoid of bright $8 \mu \mathrm{m}$ emission. The contours show the $\left(H-K_{\mathrm{S}}\right)$ color distribution. The solid and dashed contours correspond to 0.52 and $0.68 \mathrm{mag}$, respectively. The black open stars represent the target positions (Table 1) where we conducted single pointing observations. The red filled stars correspond to IRAS point sources.

using the Mann-Whitney-U-test, is performed on a $60^{\prime \prime} \times 60^{\prime \prime}$ grid covering the entire Outer Galactic Plane. Adjacent cells where the $\left(H-K_{\mathrm{S}}\right)$ color distribution of stars is different from the local surroundings on a high (>99\%) confidence level are selected as initial targets. The dark cloud candidates are then chosen on the basis of their abnormal red color and an absence of counterparts in SIMBAD. In this section we briefly describe the appearance of the G111.80+0.58 region in MSX, 2MASS and FCRAO data. Details of interest for the individual target positions which were selected for single pointing observations (see Sect. 4.3) are discussed in Sect. 6.5.

We selected this region specifically for a follow-up study because it was identified as an excessively red, filamentary region in a pilot-survey covering blindly about ten square degrees in the second Galactic quadrant. The color scaling in Figs. 1-3 shows the MSX $8 \mu \mathrm{m}$ emission toward the location of the complex and its immediate surroundings. The two prominent emission regions labeled A and B correspond to NGC 7538 and S159, respectively. The weaker emission near label $\mathrm{C}$ is associated with several masers and radio sources. The outlined region depicts the area mapped in $\mathrm{C}^{18} \mathrm{O}$ (Fig. 4). The target positions for single pointing observations (Table 1) are indicated by the black open stars. Compared to the active regions in the field, only faint $8 \mu \mathrm{m}$ emission $\left(<5 \times 10^{-6} \mathrm{Wm}^{-2} \mathrm{sr}^{-1}\right)$ is associated with this area. Within the box, several IRAS sources are present, indicated by the filled red stars.

The blue contours in Fig. 1 display the red tail of the $\left(H-K_{\mathrm{S}}\right)$ colors of stars observed in 2MASS on an oversampled $30^{\prime \prime} \times 30^{\prime \prime}$ grid. The solid and dashed contour represent the values 0.52 and $0.68 \mathrm{mag}$, respectively. Assuming that the $\left(H-K_{\mathrm{S}}\right)$ colors are related to the extinction they can be analyzed 


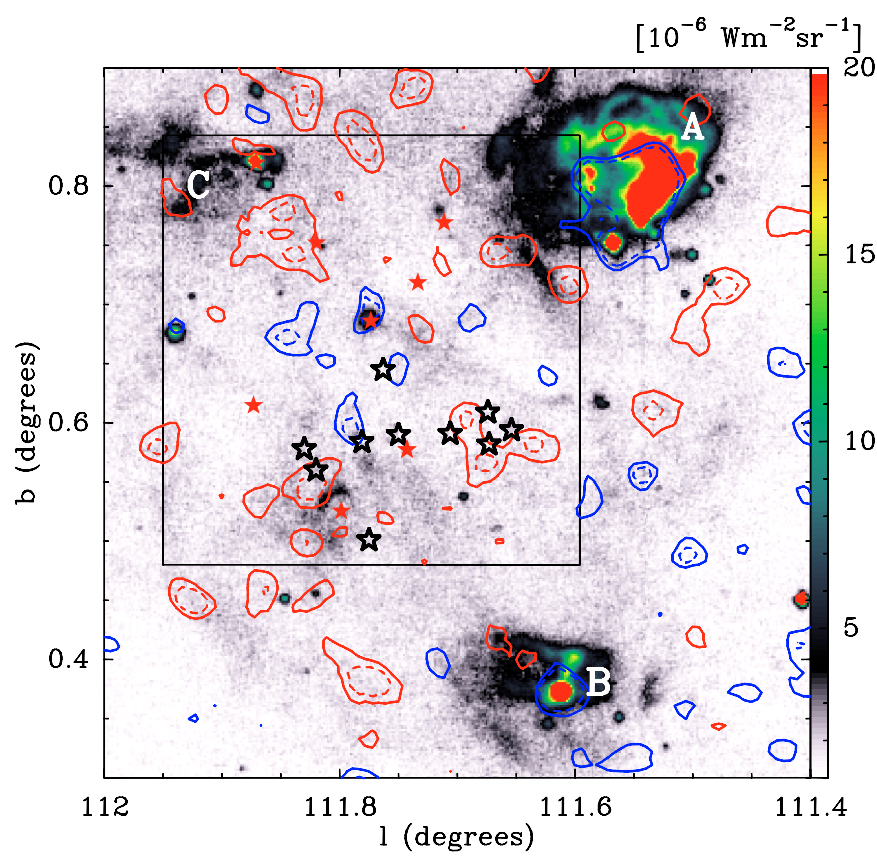

Fig. 2. Same as Fig. 1, except the contours represent the 2MASS source distribution on a $60^{\prime \prime} \times 60^{\prime \prime}$ grid. The red dashed and solid contours correspond to 3 and 4 stars per cell, respectively. The blue dashed and solid contours correspond to 13 and 11 stars per cell, respectively. The average source count per cell is 8 .

using the Near Infrared Color Excess technique (e.g., Lada et al. 1994). The near-IR extinction in a cell is then given by

$\left\langle E\left(H-K_{\mathrm{S}}\right)_{\mathrm{cell}}\right\rangle=\frac{\sum_{i=1}^{N} E\left(H-K_{\mathrm{S}}\right)_{i}}{N}$,

where the summation is over $N$ stars present in a grid-cell. The color excess $E\left(H-K_{\mathrm{S}}\right)_{i}$ per star is the difference between the observed color and the intrinsic color, where the latter is derived from an off-position chosen to represent the color distribution free of extinction. Note that foreground stars will play a significant role and reduce the average reddening per cell at large cloud distances. A correction for this, e.g., by comparing with the off-position, is required to determine the extinction accurately. This is the main reason why the identification is based on a statistical color distribution instead of the color excess, because a priori a distance to the objects is unknown and off-positions were not chosen while processing the entire Outer Galactic Plane. Keeping this in mind and adopting an intrinsic $\left(H-K_{\mathrm{S}}\right)$ value of $0.14 \mathrm{mag}$, the contour values correspond to $\sim 6$ and $\sim 8.5$ mag extinction, for convenience converted to $A_{\mathrm{V}}$ using a standard extinction law (i.e., $A_{\mathrm{V}}=15.9 \times\left\langle E\left(H-K_{\mathrm{S}}\right)_{\text {cell }}\right\rangle$; Rieke \& Lebofsky 1985). The peak extinction that is measured this way corresponds to $\sim 15 \mathrm{mag}$ in $A_{\mathrm{V}}$. Note that these values are similar to those reported for Inner Galaxy IRDCs using the same color excess method (e.g., G48 toward W51 with peak $A_{\mathrm{V}} \sim 10-20$ mag; Shipman et al. 2003).

The contours in Fig. 2 give a measure of the 2MASS stellar distribution. The mean distribution of the 2 by 2 square degree surrounding field is $\sim 8$ stars per $60^{\prime \prime} \times 60^{\prime \prime}$ cell with a $1 \sigma$ spread of 3 stars. The red contours depict the regions deficit in stars (4 and 3 stars per cell) whereas the blue contours show a surplus (11 and 13 stars per cell). The star counts are also related to the extinction along the line of sight, but here, a direct translation is difficult because they represent data from an incomplete 2MASS catalog, i.e., the Point Source Catalog including the faint extension. Therefore, they merely give an indication

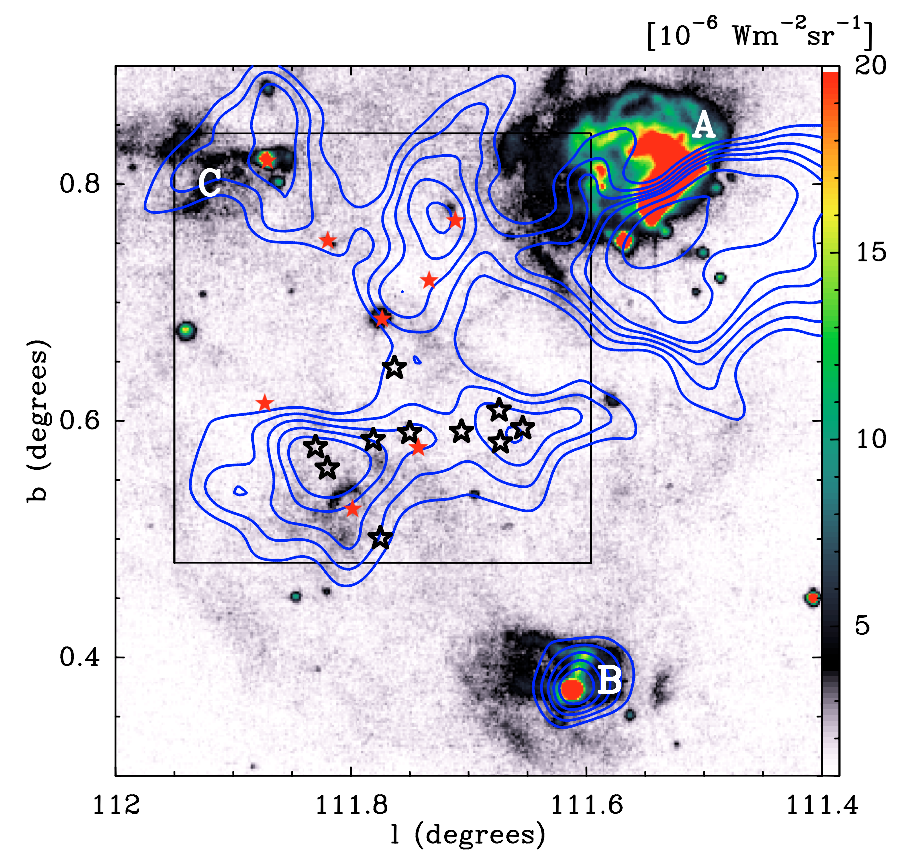

Fig. 3. Same as Fig. 1, except the contours represent the integrated $\mathrm{CO}$ emission between -45 and $-60 \mathrm{~km} \mathrm{~s}^{-1}$ as observed in the FCRAO survey. The contours range from 60 to $100 \mathrm{~K} \mathrm{~km} \mathrm{~s}^{-1}$ in steps of $10 \mathrm{~K} \mathrm{~km} \mathrm{~s}^{-1}$.

of the stellar distribution and the number of stars that is used for the identification and color excess method.

Based on the 2MASS data, four different appearances can be distinguished in the area of interest and may be explained intuitively by the following:

1) The combination of color excess and a deficit in star counts. The measured extinction is due to background and, if present, embedded stars and the column of foreground material is large resulting in fewer background stars compared to the off-position.

2) The combination of color excess and a surplus in star counts. The measured extinction is due to background and embedded sources. The embedded objects result in an enhancement in the star count distribution.

3) Only star count contours. A large column of material prevents the observation of sufficient background reddened stars resulting in a decline of the stellar distribution. The average color distribution is normal either due to a complete absence of red background stars or due to the presence of too many foreground stars.

4) Only color excess contours. Red background and embedded sources account for the average red color and the embedded sources may compensate for the deficit of background stars due to the extinction.

Note that S159 and NGC 7538 both show up as reddened regions and have a surplus of stars, as can be expected for these active star forming regions where multiple embedded sources are present.

G111.80+0.58 is not an entirely unknown object. It was identified in the FCRAO survey and cataloged as part of a molecular cloud (Brunt et al. 2003). The blue contours in Fig. 3 display the $\mathrm{CO}$ data integrated approximately between -45 and $-60 \mathrm{~km} \mathrm{~s}^{-1}$. The cloud is in velocity space as well as in projection on the sky part of a large star forming molecular cloud complex in the Perseus spiral arm which also includes the aforementioned regions NGC 7538 and S159. The kinematic distance 
Table 1. Source list.

\begin{tabular}{cccc}
\hline \hline Name & $\begin{array}{c}l \\
\left({ }^{\circ}\right)\end{array}$ & $\begin{array}{c}b \\
\left(^{\circ}\right)\end{array}$ & $\begin{array}{c}S^{a} \\
\left(\frac{D}{3.1} \mathrm{pc}\right)\end{array}$ \\
\hline G111.80+0.58 & 111.80 & 0.575 & \\
P1 & 111.65 & 0.59 & 1.04 \\
P2 & 111.67 & 0.58 & 0.80 \\
P3 & 111.67 & 0.61 & 1.14 \\
P4 & 111.71 & 0.59 & 0.77 \\
P5 & 111.75 & 0.59 & 1.01 \\
P6 & 111.76 & 0.65 & 1.01 \\
P7 & 111.78 & 0.50 & 0.90 \\
P8 & 111.78 & 0.58 & 1.19 \\
P9 & 111.82 & 0.56 & 0.60 \\
P10 & 111.83 & 0.58 & 1.13 \\
S159 (reference) & 111.61 & 0.38 & 0.60 \\
\hline
\end{tabular}

a Size of the corresponding core (see Sect. 5.2), derived from the $\mathrm{C}^{18} \mathrm{O}$ map displayed in Fig. 4 and assuming a distance $D$ of $3.1 \mathrm{kpc}$, where $D$ is adopted from S159 (Brand \& Blitz 1993).

to the G111.80+0.58 complex, assuming a flat rotation curve and the IAU standard constants $V_{\odot}=220 \mathrm{~km} \mathrm{~s}^{-1}$ and $R_{\odot}=8.5 \mathrm{kpc}$, is $\sim 5.0 \mathrm{kpc}$. S159 has a similar central velocity $\left(-56 \mathrm{~km} \mathrm{~s}^{-1}\right)$ as the object presented in this paper. However, a distance of $3.1 \mathrm{kpc}$ was reported by Brand \& Blitz (1993) for this cloud, based on spectrophotometric observations of associated stars. Also, the distance to NGC 7538 is generally considered to be less $(2.8 \mathrm{kpc}$; e.g., van der Tak et al. 2000) than the kinematic distance. It appears that this part of the Perseus spiral arm is actually closer to us than suggested by the radial velocity. For the analysis in this paper we adopt a distance of $3.1 \pm 0.2 \mathrm{kpc}$, but this may be scaled accordingly.

More extensive details on the identification of candidate IRDCs in the Outer Galactic Plane are given in FS07, but the spectroscopic data presented in this paper indeed confirm the presence of a filamentary molecular cloud complex at the identified position.

\section{Observations}

The observations presented in this paper were carried out with the Effelsberg 100-m telescope in Germany and the IRAM 30-m telescope in Spain early 2005. An overview of the observed positions and the main parameters of the observed lines are given in Tables 1 and 2.

\subsection{Effelsberg 100-m observations}

Single pointing observations of the $\mathrm{NH}_{3}(1,1),(2,2)$ and $(3,3)$ inversion transitions were performed using the Effelsberg $100-\mathrm{m}$ telescope of the Max-Planck-Institut für Radioastronomie to assess the gas kinetic temperatures at the positions listed in Table 1. The first set of data were taken under reasonable winter conditions in January and February 2005 using the $18-26 \mathrm{GHz}$ frontend in position switch mode. The receiver was tuned to a central frequency of $22 \mathrm{GHz}$ and the auto-correlator with 2 subunits of $20 \mathrm{MHz}$ bandwidth covered the 3 transitions simultaneously. After smoothing the data the spectral resolution was $\approx 0.2 \mathrm{~km} \mathrm{~s}^{-1}$. At the 100 -m telescope, no chopper wheel calibration is available, so that the atmospherical opacity and the gain response of the receiver need to be calibrated with photometric references. The details of the procedure used to calibrate our data are given in Appendix A. The resulting system noise temperatures are of order $120-400 \mathrm{~K}$. A beam efficiency of $\eta=0.58$ is used to convert the antenna temperature $T_{\mathrm{A}}^{*}$ to the main beam temperature $T_{\mathrm{mb}}$. Additional data were taken in the following
Table 2. Line parameters.

\begin{tabular}{ccccccc}
\hline \hline Line & $\begin{array}{c}\text { Frequency } \\
(\mathrm{GHz})\end{array}$ & $\begin{array}{c}\mathrm{HPBW} \\
\left({ }^{\prime \prime}\right)\end{array}$ & $\begin{array}{c}T_{\text {sys }} \\
(\mathrm{K})\end{array}$ & $F_{\text {eff }}{ }^{a}$ & $B_{\text {eff }}{ }^{b}$ \\
\hline${ }^{13} \mathrm{CO}$ & $1-0$ & 110.201 & 22.5 & $100-200$ & 0.95 & 0.75 \\
& $2-1$ & 220.399 & 11.2 & $400-900$ & 0.91 & 0.77 \\
$\mathrm{C}^{18} \mathrm{O}$ & $1-0$ & 109.782 & 22.5 & $110-200$ & 0.95 & 0.75 \\
& $2-1$ & 219.560 & 11.2 & $200-600$ & 0.91 & 0.77 \\
$\mathrm{C}^{34} \mathrm{~S}$ & $2-1$ & 96.413 & 25.5 & $115-135$ & 0.95 & 0.55 \\
$\mathrm{NH}_{3}$ & 1,1 & 23.694 & 40 & $120-400$ & - & 0.58 \\
& 2,2 & 23.723 & 40 & $120-400$ & - & 0.58 \\
& 3,3 & 23.870 & 40 & $120-400$ & - & 0.58 \\
\hline
\end{tabular}

${ }^{a}$ Forward efficiency, only for IRAM observations.

${ }^{b}$ Beam efficiency.

${ }^{c} 200-350 \mathrm{~K}$ for the map, $400-600 \mathrm{~K}$ for single pointing observations.

months and calibration was performed using a position observed in the first run. Data reduction was done using the CLASS package (Forveille et al. 1989). For the analysis of the kinetic temperature the absolute scaling is not a concern because it depends only on the ratio of the hyperfine $(1,1)$ lines and the $(1,1)$ to $(2,2)$ brightness temperature ratios. The spectra are displayed in Figs. B.1-B.3 in the Appendix.

\subsection{IRAM 30-m observations}

Observations at the IRAM-30 m telescope were conducted under excellent winter conditions (zenith opacity at $220 \mathrm{GHz} \lesssim 0.05$ ) in February 2005. A fully-sampled map of $18^{\prime} \times 18^{\prime}$ in $\mathrm{C}^{18} \mathrm{O}$ $2-1$, covering the main complex, was obtained using the HERA multi-beam instrument (Schuster et al. 2004). The corresponding SSB receiver and system temperatures were $\approx 100-250 \mathrm{~K}$ and $\approx 200-350 \mathrm{~K}$, respectively. The on-the-fly observing mode was used to allow continuous data acquisition as the antenna was moving, with a scanning velocity of $1^{\prime \prime} \mathrm{s}^{-1}$. Frequency-switching mode was used to subtract the sky background contribution. The HERA matrix projected on the sky was rotated by $9.6^{\circ}$ to provide a $4^{\prime \prime}$ spatial sampling in both directions. Maps were done in lambda and beta directions to minimize striping effects due to temporal drifts. Each spectrum has 896 channels of $80 \mathrm{kHz}$ or $0.1 \mathrm{~km} \mathrm{~s}^{-1}$ width thanks to the VESPA autocorrelator facility backend. Data were reduced using the GILDAS software ${ }^{1}$. The bandpass of the system was removed from each spectrum prior to folding, by subtracting a low-order $(\leq 3)$ polynomial. The spectra were then resampled on a $6^{\prime \prime}$ grid by Gaussian convolution with a final rms of $0.17 \mathrm{~K}$ in each $0.1 \mathrm{~km} \mathrm{~s}^{-1}$ channel.

In addition, pointing observations were performed toward the integrated intensity peaks in $\mathrm{C}^{18} \mathrm{O} 1-0$ and 2-1 (for column density), ${ }^{13} \mathrm{CO} 1-0$ and 2-1 (for temperature) and $\mathrm{C}^{34} \mathrm{~S} 2-1$ (as a high density tracer). The receivers were connected to an autocorrelator with a resolution of $80 \mathrm{kHz}$ resulting in velocity channels of $0.2-0.25$ and $0.1 \mathrm{~km} \mathrm{~s}^{-1}$ at 3 and $1.3 \mathrm{~mm}$, respectively. The rms noise levels are of the order of 0.3 and $0.5 \mathrm{~K}$ at 3 and $1.3 \mathrm{~mm}$, respectively. The absolute calibration to obtain the main beam temperature $T_{\mathrm{mb}}$ involved a standard conversion of the antenna temperature $T_{\mathrm{A}}^{*}$ using the forward and beam efficiencies listed in Table 2. The observed intensities, and hence all the derived parameters, are overestimated due to a contribution of the emission present in the error beam. We adopt a very conservative estimate for this contribution of $20 \%$.

${ }^{1}$ URL: http://www.iram.fr/IRAMFR/GILDAS/ 


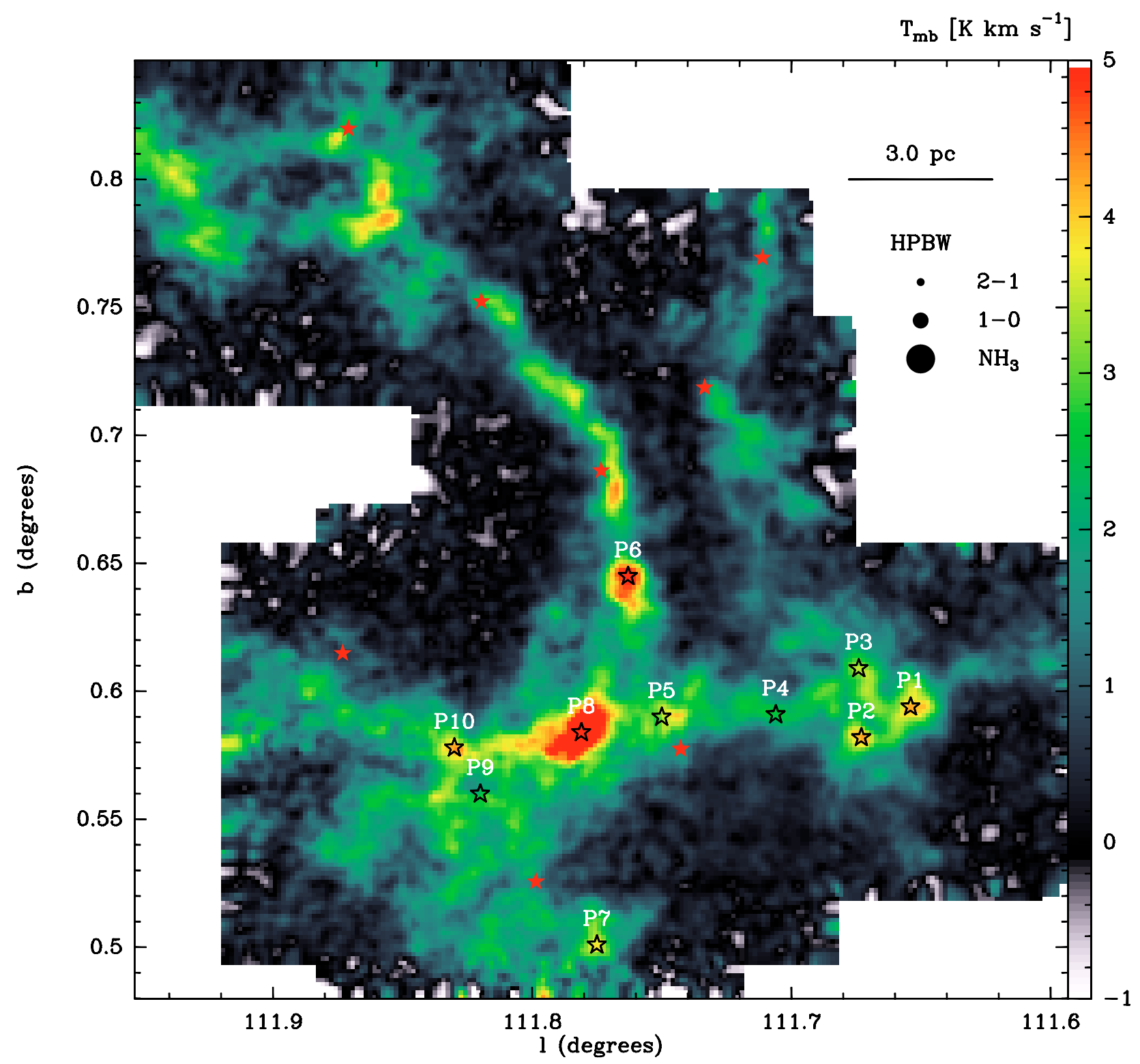

Fig. 4. This figure shows the $\mathrm{C}^{18} \mathrm{O} 2-1$ map of the filamentary dark cloud complex G111.80+0.58 observed with the HERA instrument on IRAM, integrated between -56 and $-46 \mathrm{~km} \mathrm{~s}^{-1}$. The cores listed in Table 1 are given and correspond to the positions indicated by the open black stars, where additional single pointing observations were conducted. The filled red stars represent the IRAS point sources. The linear scale is indicated assuming a distance of $3.1 \mathrm{kpc}$. The approximate half-power-beam-widths (HPBW) represent the ${ }^{13} \mathrm{CO}$ and $\mathrm{C}^{18} \mathrm{O} 2-1$, the $\mathrm{C}^{34} \mathrm{~S} 2-1$, ${ }^{13} \mathrm{CO}$ and $\mathrm{C}^{18} \mathrm{O} 1-0$ and the $\mathrm{NH}_{3}$ beams, respectively.

\section{Observational properties}

\subsection{Spatial distribution: $C^{18}$ O 2-1 map}

The high-resolution $\left(\sim 12^{\prime \prime}\right)$ intensity map of the $\mathrm{C}^{18} \mathrm{O} 2-1$ transition (integrated between -48 and $-56 \mathrm{~km} \mathrm{~s}^{-1}$ ) is displayed in Fig. 4. The $\mathrm{C}^{18} \mathrm{O}$ molecule, being optically thin under typical ISM conditions, traces the gas column along the line of sight and reveals the filamentary structure in great detail. Several high column density regions are identified along the filaments and a number of these were selected for single pointing observations $(\mathrm{P} 1-\mathrm{P} 10)$. The extend of the $\mathrm{C}^{18} \mathrm{O}$ emission in the map is used to determine the size of the cores (Sect. 5.2).

The reddening contours derived from 2MASS in Fig. 1 show a similar structure, but they do not trace the $\mathrm{C}^{18} \mathrm{O}$ everywhere. This can be explained by both a lower resolution and the limitations of the color excess method, which relies completely on the presence of background and embedded red objects along the line of sight. If the extinction is too high, no red background sources are detected and the method fails. However, in such a case, a decrease in star counts (Fig. 2) can be expected and indeed this is seen for the regions near P1, P2, P3 and P4.

Filamentary structure is frequently observed in IRDCs (e.g., Carey et al. 1998; Johnstone et al. 2003) and is predicted by theories of cloud evolution and clump formation (e.g., Klessen et al. 2004).

In this paper we focus on the physical properties of a number of $\mathrm{C}^{18} \mathrm{O}$ intensity peaks, indicated by the open stars and corresponding to the positions listed in Table 1. The filled red stars represent the location of IRAS point sources in the region. Except for P5 and possibly P9 and P10 (see Sect. 6.5), the IRAS sources do not coincide or appear to interact with any of the target intensity peaks and though they may be associated with the 


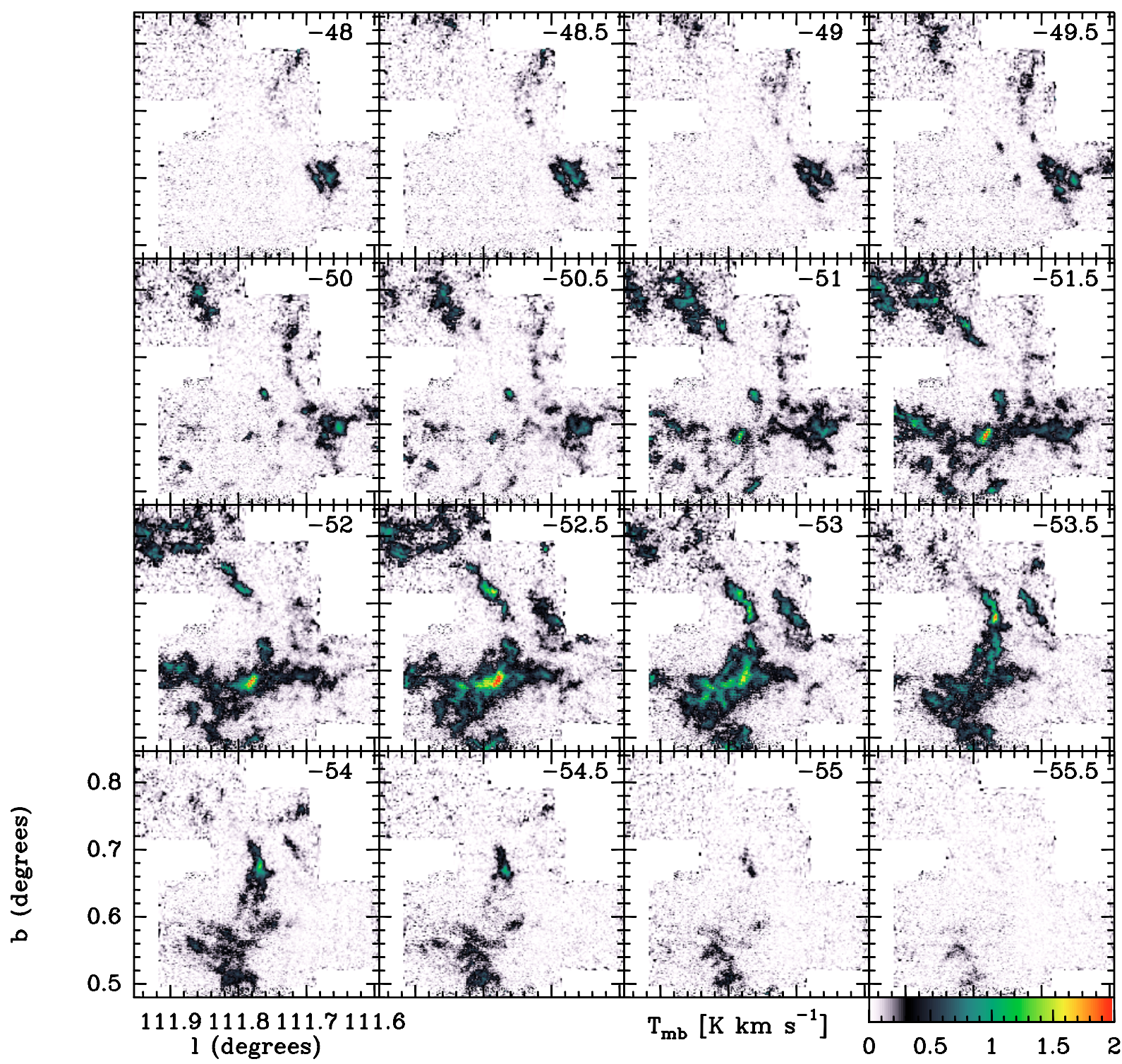

Fig. 5. This figure displays the velocity channels centered on the velocities given in the upper right corners in the range between -48 and $-55.5 \mathrm{~km} \mathrm{~s}^{-1}$ and integrated over $0.5 \mathrm{~km} \mathrm{~s}^{-1}$. The region shows a complex velocity distribution and several main features can be identified (see text).

complex, their connection is not considered further in the work presented here.

\subsection{Velocity distribution: $\mathrm{C}^{18} \mathrm{O} 2-1$ channel maps}

Though it is beyond the scope of this paper to fully analyze the velocity maps of the region it is worth showing the spectral velocity channels to reveal the complexity and different components present in the region. The velocity maps are given in Fig. 5 and below is a list of characteristic features that can be identified:

a) the horizontal structure just below the center (e.g., at $-51.5 \mathrm{~km} \mathrm{~s}^{-1}$ ) containing several of the observed positions (P4, P5, P8, P10);

$b)$ the curved, vertical structure (e.g., at $-53.5 \mathrm{~km} \mathrm{~s}^{-1}$ ) containing position $\mathrm{P} 6$ and moving up at nearer velocities;

c) the main intensity peak (corresponding to P8), possibly enhanced in intensity because of the superposition of the filamentary structures mentioned above, though the core itself is seen already at $-51 \mathrm{~km} \mathrm{~s}^{-1}$; d) the positions of $\mathrm{P} 1, \mathrm{P} 2$ and $\mathrm{P} 3$ on the right side are dominant at the nearest velocities $\left(-48\right.$ to $\left.-51 \mathrm{~km} \mathrm{~s}^{-1}\right)$ and appear to be the end of the horizontal structure, bending towards us;

$e)$ the structures in the upper left corner, where no target positions are located, may be connected with the vertical curved structure.

\subsection{Single pointings}

The observed spectra of ${ }^{13} \mathrm{CO}, \mathrm{C}^{18} \mathrm{O}$ and $\mathrm{C}^{34} \mathrm{~S}$ toward the main $\mathrm{C}^{18} \mathrm{O} 2-1$ intensity peaks in the map are given in Appendix B. Furthermore, the Gaussian components that were fitted using the GILDAS software are displayed as dashed lines. Some positions have additional components in the $\mathrm{CO}$ lines that do not correspond to a $\mathrm{C}^{34} \mathrm{~S}$ detection. The focus of this paper however, is on dense cores, i.e., cores that do have a $\mathrm{C}^{34} \mathrm{~S}$ detection. Therefore, the apparently lower density components, likely associated with lower density cores, cloud envelopes or specific phenomena such as outflows are not included in the analysis. 
Table 3. Physical properties.

\begin{tabular}{|c|c|c|c|c|c|c|c|c|c|c|}
\hline \multirow{4}{*}{ (a) } & \multirow[t]{3}{*}{ ID } & \multirow[t]{3}{*}{$\mathrm{CP}$} & \multirow[t]{2}{*}{$T_{\text {kin }}{ }^{13} \mathrm{CO}^{a}$} & \multicolumn{4}{|c|}{$N_{\mathrm{mol}}\left[\tau_{\mathrm{c}}\right]$} & \multirow{4}{*}{$\begin{array}{c}N_{\mathrm{H}_{2}} \\
\left(\frac{1.67 \times 10^{6}}{A} \times\right. \\
\left.10^{21} \mathrm{~cm}^{-2}\right) \\
(6)\end{array}$} & \multirow{4}{*}{$\begin{array}{c}n_{\mathrm{H}_{2}} \\
\left(\frac{3.1 \mathrm{kpc}}{D} \times\right. \\
\left.10^{2} \mathrm{~cm}^{-3}\right) \\
(7)\end{array}$} & \multirow{4}{*}{$\begin{array}{c}M_{\mathrm{LTE}} \\
\left(\frac{D^{2}}{9.6 \mathrm{kpc}^{2}} M_{\odot}\right) \\
(8)\end{array}$} \\
\hline & & & & \multicolumn{2}{|c|}{${ }^{13} \mathrm{CO}(1-0)$} & \multicolumn{2}{|c|}{$\mathrm{C}^{18} \mathrm{O}(1-0)$} & & & \\
\hline & & & $(\mathrm{K})$ & $\left(10^{14} \mathrm{~cm}^{-2}\right)$ & & $\left(10^{14} \mathrm{~cm}^{-2}\right)$ & & & & \\
\hline & (1) & (2) & (3) & (4) & & (5) & & & & \\
\hline & S159 & 2 & $29.1( \pm 5.1)$ & $1340( \pm 751)$ & [1.27] & $97( \pm 31)$ & [0.09] & $58( \pm 19)$ & $313( \pm 100)$ & $204( \pm 65)$ \\
\hline & P1 & 2 & $10.6( \pm 1.6)$ & $>496$ & {$[>4.79]$} & $11( \pm 4)$ & [0.11] & $6( \pm 3)$ & $20( \pm 8)$ & $67( \pm 28)$ \\
\hline & P1 & 3 & $9.8( \pm 1.4)$ & $125( \pm 29)$ & {$[1.44]$} & $45( \pm 10)$ & [0.41] & $27( \pm 6)$ & $85( \pm 18)$ & $287( \pm 62)$ \\
\hline & $\mathrm{P} 2$ & 2 & $6.8( \pm 0.9)$ & $>195$ & {$[>2.88]$} & $23( \pm 5)$ & [0.39] & $14( \pm 3)$ & $56( \pm 13)$ & $87( \pm 20)$ \\
\hline & P2 & 3 & $10.2( \pm 1.5)$ & $589( \pm 228)$ & {$[4.38]$} & $24( \pm 6)$ & {$[0.22]$} & $15( \pm 4)$ & $59( \pm 16)$ & $91( \pm 24)$ \\
\hline & P3 & 1 & $8.7( \pm 1.3)$ & $107( \pm 26)$ & {$[2.02]$} & $9( \pm 4)$ & {$[0.14]$} & $5( \pm 2)$ & $15( \pm 6)$ & $66( \pm 29)$ \\
\hline & P3 & 2 & $13.1( \pm 2.1)$ & $656( \pm 547)$ & [4.51] & $38( \pm 9)$ & {$[0.20]$} & $23( \pm 5)$ & $65( \pm 15)$ & $291( \pm 67)$ \\
\hline & P4 & 1 & $7.5( \pm 1.0)$ & $51( \pm 13)$ & {$[1.30]$} & $16( \pm 4)$ & {$[0.27]$} & $10( \pm 3)$ & $41( \pm 11)$ & $57( \pm 15)$ \\
\hline & P4 & 2 & $10.0( \pm 1.4)$ & $>488$ & {$[>3.68]$} & $21( \pm 5)$ & [0.18] & $13( \pm 3)$ & $53( \pm 13)$ & $73( \pm 18)$ \\
\hline & P5 & 2 & $9.7( \pm 1.4)$ & $227( \pm 36)$ & {$[1.84]$} & $26( \pm 5)$ & {$[0.19]$} & $15( \pm 3)$ & $50( \pm 10)$ & $154( \pm 31)$ \\
\hline & P6 & 1 & $12.4( \pm 1.9)$ & $168( \pm 53)$ & {$[1.54]$} & $17( \pm 7)$ & {$[0.15]$} & $10( \pm 4)$ & $32( \pm 13)$ & $99( \pm 41)$ \\
\hline & P6 & 2 & $12.1( \pm 1.9)$ & $>655$ & {$[>4.60]$} & $48( \pm 10)$ & {$[0.28]$} & $29( \pm 6)$ & $93( \pm 20)$ & $289( \pm 62)$ \\
\hline & P7 & 2 & $13.2( \pm 2.1)$ & $199( \pm 57)$ & {$[1.24]$} & $24( \pm 7)$ & [0.14] & $14( \pm 4)$ & $52( \pm 16)$ & $114( \pm 34)$ \\
\hline & P7 & 3 & $10.2( \pm 1.5)$ & $>318$ & {$[>5.13]$} & $18( \pm 8)$ & [0.28] & $11( \pm 5)$ & $39( \pm 17)$ & $86( \pm 38)$ \\
\hline & P8 & 2 & $13.9( \pm 2.4)$ & $>625$ & [>3.69] & $124( \pm 26)$ & [0.54] & $74( \pm 16)$ & $202( \pm 43)$ & $1025( \pm 217)$ \\
\hline & P9 & 2 & $9.4( \pm 1.3)$ & $67( \pm 18)$ & {$[1.00]$} & $10( \pm 3)$ & [0.11] & $6( \pm 2)$ & $33( \pm 11)$ & $22( \pm 7)$ \\
\hline & P9 & 3 & $13.0( \pm 1.9)$ & $250( \pm 88)$ & [2.29] & $11( \pm 5)$ & {$[0.10]$} & $6( \pm 3)$ & $34( \pm 17)$ & $22( \pm 11)$ \\
\hline & $\mathrm{P} 10$ & 3 & $11.2( \pm 2.0)$ & $>389$ & [>2.29] & $46( \pm 11)$ & {$[0.36]$} & $28( \pm 7)$ & $80( \pm 20)$ & $347( \pm 85)$ \\
\hline$(b)^{b}$ & & & $\begin{array}{c}T_{\text {kin }}\left(\mathrm{NH}_{3}\right) \\
(\mathrm{K})\end{array}$ & & & & & & & \\
\hline & S159 & 2 & $29.3( \pm 3.4)$ & $1341( \pm 742)$ & [1.25] & $97( \pm 25)$ & [0.09] & $58( \pm 15)$ & $315( \pm 82)$ & $205( \pm 54)$ \\
\hline & $\mathrm{P} 1$ & 2 & $14.4( \pm 2.6)$ & $434( \pm 108)$ & {$[1.50]$} & $12( \pm 6)$ & {$[0.07]$} & $7( \pm 4)$ & $23( \pm 11)$ & $76( \pm 38)$ \\
\hline & P2 & 2 & $19.0( \pm 8.4)$ & $149( \pm 39)$ & {$[0.36]$} & $30( \pm 20)$ & [0.08] & $18( \pm 12)$ & $73( \pm 48)$ & $113( \pm 74)$ \\
\hline & P5 & 2 & $18.2( \pm 2.7)$ & $217( \pm 46)$ & {$[0.45]$} & $34( \pm 9)$ & {$[0.08]$} & $20( \pm 5)$ & $65( \pm 17)$ & $201( \pm 53)$ \\
\hline & P8 & 2 & $16.7( \pm 1.4)$ & $470( \pm 159)$ & [1.63] & $131( \pm 29)$ & {$[0.40]$} & $79( \pm 17)$ & $215( \pm 47)$ & $1090( \pm 240)$ \\
\hline & P9 & 2 & $20.0( \pm 2.3)$ & $78( \pm 27)$ & {$[0.26]$} & $15( \pm 7)$ & [0.04] & $9( \pm 4)$ & $47( \pm 24)$ & $31( \pm 16)$ \\
\hline & P9 & 3 & $18.0( \pm 2.6)$ & $219( \pm 92)$ & {$[0.90]$} & $13( \pm 8)$ & {$[0.06]$} & $8( \pm 5)$ & $41( \pm 26)$ & $26( \pm 17)$ \\
\hline & P10 & 3 & $13.5( \pm 2.1)$ & $435( \pm 141)$ & [2.68] & $49( \pm 13)$ & [0.07] & $29( \pm 8)$ & $84( \pm 22)$ & $366( \pm 94)$ \\
\hline
\end{tabular}

${ }^{a}$ The kinetic temperature is assumed equal to the excitation temperature.

${ }^{b}$ Part $b$ is the same as part $a$, except here the kinetic temperature is derived from $\mathrm{NH}_{3}$. Note that the results are very similar.

We used the following approach to define the dense components; 1) Gauss-fitting the $\mathrm{C}^{34} \mathrm{~S}$ with $N$ components, 2) Gaussfitting the $\mathrm{C}^{18} \mathrm{O}$ and ${ }^{13} \mathrm{CO}$ species with at least $N$ lines, 3 ) imposing as constraints the positions that correspond to the $\mathrm{C}^{34} \mathrm{~S}$ fits. The different components are numbered according to increasing central velocity (Col. 2, Table 3 ). Some of the ${ }^{13} \mathrm{CO}$ lines show high optical depth effects and are impossible to match in velocity with the optically thin lines. In that case fits are produced with the central velocity as a free parameter. Hence the $\mathrm{C}^{18} \mathrm{O}$ is used to determine the column densities and masses.

\section{Results: physical properties of the clouds}

The physical characteristics of the cores that are determined from the molecular lines are listed in Tables 3 and 4. The integrated properties (column density and derived parameters) as well as the ${ }^{13} \mathrm{CO}$ temperature are determined by using the Gaussian fits of the individual components. A brief description of the tables and their content is given here. More details on the derivation of the values are presented in the following sub-sections:

Table 3-a) Physical properties assuming the kinetic temperature given by ${ }^{13} \mathrm{CO}$.

Column (1): Identification number of the cores sorted according to Galactic coordinates.
Column (2): Identification number $n$ of the component along the line of sight; corresponds to the $n^{\text {th }}$ component in the spectra counting from the left, where $n$ is the same for the different molecules if they have a similar central velocity. Note that only those components that correspond to $\mathrm{C}^{34} \mathrm{~S}$ emission are part of the analysis and only these are listed.

Column (3): Kinetic temperature $T_{\text {kin }}(\mathrm{K})$ derived from ${ }^{13} \mathrm{CO}$. Columns $(4,5)$ : Column density (in units of $10^{14} \mathrm{~cm}^{-2}$ ) of the CO molecules assuming LTE and optically thin emission. In brackets the optical depth $\tau_{\mathrm{c}}$ at the line center is given. Note that the ${ }^{13} \mathrm{CO}$ emission is in general optically thick in dense cores and for some positions only a lower limit could be determined for both $\tau_{\mathrm{c}}$ and $N_{\mathrm{mol}}$.

Column (6): Column density (in units of $10^{21} \mathrm{~cm}^{-2}$ ) of $\mathrm{H}_{2}$ derived from the $\mathrm{C}^{18} \mathrm{O}$ emission using a standard abundance of $1.67 \times 10^{-7}$ (Frerking et al. 1982).

Column (7): Average $\mathrm{H}_{2}$ volume density (in units of $10^{2} \mathrm{~cm}^{-3}$ ) derived from the $\mathrm{H}_{2}$ column and using the size.

Column (8): LTE Mass $\left(M_{\odot}\right)$ given by Eq. (9) assuming a constant volume density and using the size from Table 1.

Table 3-b) Physical properties assuming the kinetic temperature given by $\mathrm{NH}_{3}$.

Columns (1,2): Same as part $a$.

Column (3): Kinetic temperature $T_{\text {kin }}(\mathrm{K})$ derived from $\mathrm{NH}_{3}$.

Columns (4-8): Same as part $a$. 
Table 4. Line-width and mass properties.

\begin{tabular}{|c|c|c|c|c|c|c|c|c|c|}
\hline \multirow[t]{3}{*}{ ID } & \multirow[t]{3}{*}{$\mathrm{CP}$} & \multicolumn{4}{|c|}{$\Delta V$} & $M_{\text {vir }}$ & $M_{\mathrm{LTE}}$ & $M_{\text {Jeans }}$ & $\eta$ \\
\hline & & ${ }^{13} \mathrm{CO}$ & $\mathrm{C}^{18} \mathrm{O}$ & $\mathrm{C}^{34} \mathrm{~S}$ & virial & & & & \\
\hline & & $\left(\mathrm{km} \mathrm{s}^{-1}\right)$ & $\left(\mathrm{km} \mathrm{s}^{-1}\right)$ & $\left(\mathrm{km} \mathrm{s}^{-1}\right)$ & $\left(\mathrm{km} \mathrm{s}^{-1}\right)$ & $\left(\frac{D}{3.1 \mathrm{kpc}} M_{\odot}\right)$ & $\left(\frac{D^{2}}{9.6 \mathrm{kpc}^{2}} M_{\odot}\right)$ & $\left(M_{\odot}\right)$ & $\frac{E_{\mathrm{T}}}{E_{\text {grav }}}$ \\
\hline (1) & (2) & (3) & (4) & (5) & (6) & (7) & (8) & (9) & (10) \\
\hline S159 & 2 & $2.84( \pm 0.01)$ & $2.40( \pm 0.02)$ & $2.25( \pm 0.04)$ & $2.36( \pm 0.02)$ & $352( \pm 59)$ & $204( \pm 65)$ & $15( \pm 5)$ & $0.09( \pm 0.03)$ \\
\hline $\mathrm{P} 1$ & 2 & $3.00( \pm 0.06)$ & $1.07( \pm 0.15)$ & $1.57( \pm 0.25)$ & $1.22( \pm 0.13)$ & $162( \pm 38)$ & $67( \pm 28)$ & $13( \pm 4)$ & $0.17( \pm 0.08)$ \\
\hline P1 & 3 & $1.77( \pm 0.08)$ & $1.74( \pm 0.05)$ & $2.27( \pm 0.50)$ & $1.77( \pm 0.06)$ & $342( \pm 40)$ & $287( \pm 62)$ & $5( \pm 1)$ & $0.04( \pm 0.01)$ \\
\hline P2 & 2 & $2.19( \pm 0.02)$ & $1.95( \pm 0.03)$ & $1.33( \pm 0.33)$ & $1.91( \pm 0.04)$ & $307( \pm 40)$ & $87( \pm 20)$ & $4( \pm 1)$ & $0.07( \pm 0.02)$ \\
\hline P2 & 3 & $3.98( \pm 0.02)$ & $1.62( \pm 0.02)$ & $1.67( \pm 0.85)$ & $1.62( \pm 0.03)$ & $220( \pm 29)$ & $91( \pm 24)$ & $7( \pm 2)$ & $0.09( \pm 0.03)$ \\
\hline P3 & 1 & $1.34( \pm 0.02)$ & $1.17( \pm 0.04)$ & $1.47( \pm 0.29)$ & $1.20( \pm 0.05)$ & $172( \pm 20)$ & $66( \pm 29)$ & $11( \pm 3)$ & $0.16( \pm 0.07)$ \\
\hline P3 & 2 & $2.91( \pm 0.02)$ & $2.07( \pm 0.03)$ & $1.06( \pm 0.34)$ & $2.02( \pm 0.03)$ & $489( \pm 46)$ & $291( \pm 67)$ & $10( \pm 3)$ & $0.05( \pm 0.01)$ \\
\hline P4 & 1 & $1.28( \pm 0.02)$ & $1.63( \pm 0.05)$ & $0.82( \pm 0.19)$ & $1.51( \pm 0.05)$ & $185( \pm 27)$ & $57( \pm 15)$ & $5( \pm 1)$ & $0.10( \pm 0.03)$ \\
\hline P4 & 2 & $2.52( \pm 0.02)$ & $1.74( \pm 0.05)$ & $1.26( \pm 0.12)$ & $1.63( \pm 0.05)$ & $215( \pm 30)$ & $73( \pm 18)$ & $7( \pm 2)$ & $0.11( \pm 0.03)$ \\
\hline P5 & 2 & $2.83( \pm 0.02)$ & $2.25( \pm 0.04)$ & $2.62( \pm 0.24)$ & $2.29( \pm 0.05)$ & $556( \pm 59)$ & $154( \pm 31)$ & $7( \pm 2)$ & $0.07( \pm 0.02)$ \\
\hline P6 & 1 & $1.49( \pm 0.01)$ & $1.14( \pm 0.03)$ & $0.96( \pm 0.16)$ & $1.12( \pm 0.03)$ & $132( \pm 15)$ & $99( \pm 41)$ & $13( \pm 4)$ & $0.13( \pm 0.06)$ \\
\hline P6 & 2 & $2.88( \pm 0.01)$ & $2.09( \pm 0.02)$ & $1.20( \pm 0.01)$ & $2.08( \pm 0.03)$ & $459( \pm 47)$ & $289( \pm 62)$ & $7( \pm 2)$ & $0.04( \pm 0.01)$ \\
\hline P7 & 2 & $1.91( \pm 0.02)$ & $1.49( \pm 0.11)$ & $1.62( \pm 0.30)$ & $1.52( \pm 0.11)$ & $218( \pm 40)$ & $114( \pm 34)$ & $11( \pm 3)$ & $0.11( \pm 0.04)$ \\
\hline P7 & 3 & $1.41( \pm 0.01)$ & $1.05( \pm 0.02)$ & $1.68( \pm 0.77)$ & $1.06( \pm 0.02)$ & $106( \pm 13)$ & $86( \pm 38)$ & $9( \pm 3)$ & $0.11( \pm 0.05)$ \\
\hline P8 & 2 & $2.32( \pm 0.02)$ & $2.22( \pm 0.01)$ & $2.08( \pm 0.05)$ & $2.20( \pm 0.01)$ & $604( \pm 51)$ & $1025( \pm 217)$ & $6( \pm 2)$ & $0.02( \pm 0.00)$ \\
\hline P9 & 2 & $1.55( \pm 0.02)$ & $1.48( \pm 0.08)$ & $1.57( \pm 0.22)$ & $1.50( \pm 0.08)$ & $142( \pm 28)$ & $22( \pm 7)$ & $8( \pm 2)$ & $0.27( \pm 0.10)$ \\
\hline P9 & 3 & $1.64( \pm 0.02)$ & $1.23( \pm 0.06)$ & $1.82( \pm 0.28)$ & $1.31( \pm 0.07)$ & $109( \pm 21)$ & $22( \pm 11)$ & $13( \pm 5)$ & $0.36( \pm 0.19)$ \\
\hline P10 & 3 & $2.22( \pm 0.71)$ & $1.60( \pm 0.02)$ & $1.99( \pm 0.08)$ & $1.66( \pm 0.02)$ & $326( \pm 30)$ & $347( \pm 85)$ & $7( \pm 2)$ & $0.04( \pm 0.01)$ \\
\hline
\end{tabular}

Table 4) Line-widths and mass characteristics derived from the IRAM data.

Columns $(1,2)$ : Same as Table 3.

Columns (3-5): Line-width (FWHM) of the observed molecules. The ${ }^{13} \mathrm{CO}$ and $\mathrm{C}^{18} \mathrm{O}$ values are an error-weighted mean of the 1-0 and 2-1 transitions.

Column (6): The error weighted mean FWHM of the optical thin $\mathrm{C}^{18} \mathrm{O}$ and $\mathrm{C}^{34} \mathrm{~S}$ lines that is used to derive the virial mass.

Column (7): Virial mass $\left(M_{\odot}\right)$ as given by Eq. (10) assuming a constant volume density $n\left(\mathrm{H}_{2}\right)$ and using the line-width $\Delta V$ given in Col. 6.

Column (8): LTE mass $\left(M_{\odot}\right)$. Same as Col. (8), Table 3-a. Listed here for comparison with the other masses.

Column (9): Thermal Jeans mass as given by Eq. (11).

Column (10): Thermal over gravitational energy ratio $\eta$ as given by Eq. (12).

\subsection{Kinetic temperatures}

The kinetic temperature of the cores can be estimated from both the $\mathrm{NH}_{3}$ and the ${ }^{13} \mathrm{CO}$ lines. In general, considering the conditions in dense cores, collisions dominate the excitation process and the levels are thermalized. In this case, the kinetic temperature equals the excitation temperature, i.e., $T_{\mathrm{kin}}=T_{\mathrm{ex}}$. A brief description of the determination of $T_{\mathrm{ex}}$ for both molecules is given below.

A comparison between the temperatures derived from $\mathrm{NH}_{3}$ and ${ }^{13} \mathrm{CO}$ can be misleading because they likely represent different regions within the cores. $\mathrm{NH}_{3}$ is a good temperature tracer and because the molecules do not freeze out onto the dust grains for densities below $\sim 10^{6} \mathrm{~cm}^{-3}$ (Bergin \& Langer 1997) they are observed even in very dense regions. In the inner, dense parts of cores the heating is due to cosmic rays and, if present, internal sources. The ${ }^{13} \mathrm{CO}$ molecule is likely depleted in dense and cold cores and it is optically thick due to its much larger abundance. Thus it traces mostly the outer parts of molecular clouds where heating is due to the interstellar radiation field penetrating the outer layers.

\subsubsection{Ammonia}

The rotational temperatures of the $\mathrm{NH}_{3}$ molecule, characterizing the level populations, were determined by fitting the main and hyperfine components of the $(1,1)$ transition and the main component of the $(2,2)$. The reduction of the $(1,1)$ transition was done using "METHOD NH3 $(1,1)$ " in CLASS to fit the hyperfine structure, whereas for the $(2,2)$ line a standard Gauss fitting procedure was sufficient. A detailed description of the standard analysis is given by Bachiller et al. (1987). The kinetic temperature is derived using the analytical expression given by Tafalla et al. (2004);

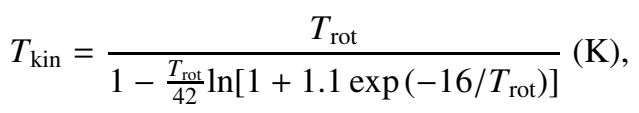

where $T_{\text {rot }}$ is the rotational temperature. Table 5 lists the properties derived from the $\mathrm{NH}_{3}$ observations. Columns 1 to 4 give the target position, line component, central velocity and line-width (FWHM) of the $(1,1)$ transition, respectively. Columns 5-6 and 9-10 give the main beam temperatures and the noise of the spectra for the $(1,1)$ and $(2,2)$ transition, respectively. The optical depth $\tau$ and the corresponding error derived from the hyperfine structure fitting for the $(1,1)$ transition are listed in Cols. 7 and 8 , respectively. Typical values for the optical depth are around unity with the exception of P1 and S159 CP2. Note however that the error is of the order of $\tau$ itself and the hyperfine fitting is considered dubious. The emission of the hyperfine structure for P2, P6 and P9 CP2, if present at all, is too weak to be detected at the observed signal to noise levels. Hence the missing entries. The rotational temperatures are given in Col. 11 with the corresponding error in Col. 12. Columns 13 and 14 give the kinetic temperatures derived using Eq. (2) and the corresponding error, respectively. Typically, the kinetic temperatures range from $13-30 \mathrm{~K}$. Note that P2 is detected only in the $(1,1)$ transition and P6 not at all, hence the missing entry of a central velocity for the latter. The $T_{\mathrm{mb}}$ values for P2 and P6 are derived from the noise of the observations and the temperature parameters merely give an upper limit. 
Table 5. $\mathrm{NH}_{3}$ derived properties.

\begin{tabular}{|c|c|c|c|c|c|c|c|c|c|c|c|c|c|}
\hline (1) & (2) & $\begin{array}{c}V_{\mathrm{LSR}} \\
\left(\mathrm{km} \mathrm{s}^{-1}\right) \\
(3)\end{array}$ & $\begin{array}{c}\Delta V \\
\left(\mathrm{~km} \mathrm{~s}^{-1}\right) \\
(4)\end{array}$ & $\begin{array}{c}T_{\mathrm{mb}}(1,1) \\
\text { (K) } \\
(5)\end{array}$ & $\begin{array}{c}E_{T_{\mathrm{mb}}(1,1)} \\
(\mathrm{K}) \\
(6)\end{array}$ & $\begin{array}{c}\overline{\tau(1,1)} \\
(7)\end{array}$ & $\begin{array}{c}E_{\tau(1,1)} \\
(8)\end{array}$ & $\begin{array}{c}T_{\mathrm{mb}}(2,2) \\
(\mathrm{K}) \\
(9)\end{array}$ & $\begin{array}{c}E_{T_{\mathrm{mb}}(2,2)} \\
(\mathrm{K}) \\
(10)\end{array}$ & $\begin{array}{l}T_{\text {rot }} \\
(\mathrm{K}) \\
\text { (11) }\end{array}$ & $\begin{array}{l}E_{T_{\text {rot }}} \\
(\mathrm{K}) \\
(12)\end{array}$ & $\begin{array}{l}T_{\text {kin }} \\
(\mathrm{K}) \\
(13)\end{array}$ & $\begin{array}{l}E_{T_{\text {kin }}} \\
(\mathrm{K}) \\
(14)\end{array}$ \\
\hline S159 & 1 & -58.01 & $1.13( \pm 0.17)$ & 0.59 & 0.06 & 1.3 & 0.7 & 0.19 & 0.05 & 17.1 & 2.0 & 18.2 & 2.0 \\
\hline$"$ & 2 & -55.87 & $2.16( \pm 0.25)$ & 0.45 & 0.06 & 0.1 & 0.1 & 0.32 & 0.05 & 25.7 & 3.4 & 29.3 & 3.4 \\
\hline $\mathrm{P} 1$ & 2 & -50.12 & $2.15( \pm 0.19)$ & 0.74 & 0.10 & 0.3 & 0.3 & 0.13 & 0.07 & 13.7 & 2.6 & 14.4 & 2.6 \\
\hline P2 & 2 & -50.95 & $1.50( \pm 0.00)$ & 0.41 & 0.20 & - & - & 0.14 & 0.14 & 17.6 & 8.4 & 19.0 & 8.4 \\
\hline P5 & 2 & -52.76 & $2.25( \pm 0.05)$ & 1.58 & 0.09 & 1.1 & 0.1 & 0.50 & 0.19 & 17.0 & 2.7 & 18.2 & 2.7 \\
\hline P6 & - & - & - & 0.12 & 0.12 & - & - & 0.08 & 0.08 & 26.4 & 23.9 & 30.2 & 23.9 \\
\hline P8 & 2 & -52.83 & $2.07( \pm 0.08)$ & 2.11 & 0.15 & 1.0 & 0.2 & 0.55 & 0.12 & 15.8 & 1.4 & 16.7 & 1.4 \\
\hline P9 & 2 & -54.18 & $2.82( \pm 0.82)$ & 0.54 & 0.06 & - & - & 0.21 & 0.05 & 18.5 & 2.3 & 20.0 & 2.3 \\
\hline$"$ & 3 & -52.03 & $1.40( \pm 0.82)$ & 0.47 & 0.06 & 1.0 & 0.1 & 0.14 & 0.05 & 16.8 & 2.6 & 18.0 & 2.6 \\
\hline P10 & 3 & -52.23 & $1.65( \pm 0.11)$ & 1.35 & 0.14 & 0.8 & 0.3 & 0.20 & 0.10 & 13.0 & 2.1 & 13.5 & 2.1 \\
\hline
\end{tabular}

\subsubsection{Optically thick ${ }^{13} \mathrm{CO}$}

From basic radiative transfer (e.g., Dickman 1978), the observed radiation temperature for a certain molecule toward a core is given by the expression

$T_{\mathrm{R}}^{*}=\left[J_{v}\left(T_{\mathrm{ex}}\right)-J_{v}\left(T_{\mathrm{bg}}\right)\right]\left[1-\exp \left(-\tau_{v}\right)\right]$,

in which

$J_{v}(T)=\frac{h v}{k} \frac{1}{\exp \left(\frac{h v}{k T}\right)-1}$.

The constants $h, k, T_{\mathrm{ex}}, T_{\mathrm{bg}}$ and $\tau_{v}$ are the Planck constant, the Boltzmann constant, the excitation temperature, the background temperature $(\sim 2.7 \mathrm{~K})$ and the optical depth at frequency $v$, respectively. In the dense cores considered in this paper, the ${ }^{13} \mathrm{CO}$ lines are usually optically thick and the results derived in Sect. 5.3 confirm indeed that $\tau_{v}$ exceeds unity at the line centers. The radiation temperature of the molecule is approximately given by

$T_{\mathrm{R}}^{*}\left({ }^{13} \mathrm{CO}\right)=\left[J_{v}\left(T_{\mathrm{ex}}\right)-J_{v}\left(T_{\mathrm{bg}}\right)\right]$,

where $T_{\mathrm{R}}^{*}\left({ }^{13} \mathrm{CO}\right)$ is the observed peak intensity of the ${ }^{13} \mathrm{CO}$ line (here we use the 1-0 transition). From this equation the excitation temperature can be derived:

$T_{\mathrm{ex}}=\frac{5.29}{\ln \left[1+\frac{5.29}{T_{\mathrm{R}}^{*}\left({ }^{13} \mathrm{CO}\right)+0.868}\right]}(\mathrm{K})$.

The values are listed in Col. (3) of Table 3-a.

In the following analysis the ${ }^{13} \mathrm{CO} 1-0$ temperature is adopted as the kinetic temperature of the clouds. When the data allow, the analysis is also performed using the kinetic temperature derived from the $\mathrm{NH}_{3}$ lines.

\subsection{Core sizes}

We define a size for each core as the average of the minor and major axis of the $50 \%$ peak intensity level observed in the $\mathrm{C}^{18} \mathrm{O}$ 2-1 map. The sizes are given in parsecs in Col. 4 of Table 1, assuming a distance to the cores of $3.1 \mathrm{kpc}$. Several positions have multiple components along the line of sight and obviously the size is then determined from a superposition of several cores. However, a distinction between the components is difficult because of the small separation in velocity so that the same size is adopted for all components. Position P9 is not identified as a coherent core in the map and a size of $40^{\prime \prime}$ is adopted. P4 is part of a filamentary structure and the size is assumed equal to the projected width of the filament.

\subsection{Column densities}

The following approach (see e.g., Bourke et al. 1997) assumes these conditions: a) the cores are isothermal, and the kinetic temperature, $T_{\text {kin }}$, is given by the excitation temperature derived from ${ }^{13} \mathrm{CO}$ or $\mathrm{NH}_{3}$; b) the cores have a constant density; c) the cores are in local thermodynamic equilibrium (LTE). Then, using the observed $\mathrm{C}^{18} \mathrm{O}$ radiation temperature $T_{\mathrm{R}}^{*}(\mathrm{~K})$, the optical depth can be calculated as a function of the LSR velocity $V$ $\left(\mathrm{km} \mathrm{s}^{-1}\right)$ parameterized by the frequency $v$ :

$$
\tau_{V}\left(\mathrm{C}^{18} \mathrm{O}\right)=-\ln \left\{1-\frac{k T_{\mathrm{R}}^{*}}{h v}\left[\frac{1}{\exp \left(\frac{h v}{k T_{\mathrm{ex}}}\right)-1}-\frac{k J_{v}\left(T_{\mathrm{bg}}\right)}{h v}\right]^{-1}\right\}
$$

The corresponding column density can be estimated using

$N\left(\mathrm{C}^{18} \mathrm{O}\right)=2.42 \times 10^{14} \sum \frac{\tau_{V}\left(\mathrm{C}^{18} \mathrm{O}\right) \Delta V T_{\mathrm{ex}}}{1-\exp \left(-\frac{h v}{k T_{\mathrm{ex}}}\right)}\left(\mathrm{cm}^{-2}\right)$,

where $h, v, k, J_{v}\left(T_{\mathrm{bg}}\right)$ are the same as before and $\Delta V$ is the velocity step size in $\mathrm{km} \mathrm{s}^{-1}$. The column densities derived for $\mathrm{C}^{18} \mathrm{O}$ 1-0 are given in Col. (5) of Table 3. The values listed in brackets corresponds to the optical depth, $\tau_{\mathrm{c}}$, at the line center and are typically much less than unity for $\mathrm{C}^{18} \mathrm{O}$.

An analogous derivation is done for the ${ }^{13} \mathrm{CO} 1-0$ transition and these values are listed in Col. (4). However, as noted before, in the dense cores the ${ }^{13} \mathrm{CO}$ is usually optically thick (i.e., $\tau_{\mathrm{c}}>1$ ) and the lines cannot be used to determine a reliable column density. Nonetheless, the results are listed and for the cases where the optical depth becomes too large, a lower limit is given. The total molecular column densities, $N\left(\mathrm{H}_{2}\right)$, are derived from the $\mathrm{C}^{18} \mathrm{O} 1-0$ results using a canonical abundance of $1.67 \times$ $10^{-7}$ (Frerking et al. 1982). These values are given in Col. 6 of Table 3.

\subsection{Volume densities}

Column 7 (Table 3) lists the volume density derived from the $\mathrm{H}_{2}$ column density, assuming the depth of the core equals the size. The density is by definition an average over the actual density profile along the line of sight. In the inner parts of the cores the density is considerably higher, as suggested by the presence of $\mathrm{C}^{34} \mathrm{~S}$. 


\subsection{Mass estimates}

\subsubsection{LTE mass}

Given the size and adopting a constant volume density, the molecular mass derived from the lines, referred to as the LTE mass, is determined using

$M_{\mathrm{LTE}}=\frac{4}{3} \pi \mu m_{\mathrm{H}} n\left(\mathrm{H}_{2}\right)\left(\frac{1}{2} S\right)^{3} \mathrm{C}\left(M_{\odot}\right)$,

where $n\left(\mathrm{H}_{2}\right)$ is the volume density, $S$ is the size, $m_{\mathrm{H}}$ is the mass of a hydrogen atom, $\mu=2.33$ is the mean molecular weight consistent with a $25 \%$ mass fraction of helium and $\mathrm{C}=1 \mathrm{pc}^{3} /$ $1 M_{\odot} \sim 1.48 \times 10^{22} \mathrm{~cm}^{3} / \mathrm{g}$, the conversion to solar masses. The values are listed in Col. 8 (Table 3 ). Because the volume density is derived from the column density $\left(n\left(\mathrm{H}_{2}\right) \sim N\left(\mathrm{H}_{2}\right) / S\right)$, the LTE mass of the cores scales with the square of the size and thus the distance (i.e., $M_{\mathrm{LTE}} \sim S^{2}$ and $\sim D^{2}$ ).

\subsubsection{Virial mass}

Comparing the LTE mass with the virial mass enables an evaluation of the dynamical state of the cores. Assuming a spherical core with uniform density and considering only thermal and dynamical broadening (neglecting e.g., magnetic fields, internal heating) the virial mass can be derived using the expression (e.g., MacLaren et al. 1988)

$M_{\mathrm{vir}}=210 R(\Delta V)^{2}\left(M_{\odot}\right)$,

where $R\left(=\frac{S}{2}\right)$ is the radius of the core in pc and $\Delta V$ is the FWHM of the line in $\mathrm{km} \mathrm{s}^{-1}$. In our case a weighted average of the observed optically thin lines, given in Col. 6 of Table 4, is used. The resulting virial masses are given in Col. 7 of Table 4 . Equation (10) assumes a constant density. The constant may be replaced by 190 or 126 for a density profile given by $\rho \sim r^{-1}$ or $\rho \sim r^{-2}$, respectively.

\subsubsection{Jeans mass}

Thermal Jeans fragmentation, often referred to as the mechanism to set the mass scale for star formation (Larson 1985), is approximately given by

$M_{\mathrm{Jeans}} \approx \frac{90}{\mu^{2}} \times T^{\frac{3}{2}} n^{-\frac{1}{2}}\left(M_{\odot}\right)$,

where $T$ is the gas temperature and $n$ is the total volume density. The values are listed in Col. 9 of Table 4 . The typical value is of the order of $10 M_{\odot}$, much smaller than the derived LTE masses of the cores. Therefore, structure on much smaller scales may be expected within the cores.

\subsubsection{Stability}

The energy ratio $\eta$ listed in Col. 10 (Table 4) is defined as the thermal energy over the gravitational energy:

$\eta=\frac{E_{\mathrm{T}}}{E_{\text {grav }}}=\frac{\frac{3}{2} \frac{M_{\mathrm{LTE}}}{\mu m_{\mathrm{H}}} k T}{\frac{3}{5} \frac{G M_{\mathrm{LTE}}^{2}}{0.5 \mathrm{~S}}}$,

where $M_{\text {LTE }}$ is given in Col. 8 (Table 4), $T$ is the kinetic temperature listed in Col. 3 (Table $3-a$ ), $S$ is the size of the core, described in Sect. 5.2, $\mu, m_{\mathrm{H}}, k$ and $G$ are the mean molecular weight $(=2.33)$, the mass of a hydrogen atom, the Boltzmann constant and the gravitational constant, respectively. Typically, $\eta$ is much less than unity and it suggests that the thermal energy by itself is insufficient to support the cores against gravitational collapse.

\section{Discussion and conclusion}

What kind of object is $\mathrm{G} 111.80+0.58$ ?

Before we attempt to answer this question it is worthwhile to discuss some key properties derived from the observations and compare the results with other studies.

\subsection{Gas temperature}

The gas temperatures derived from $\mathrm{NH}_{3}(13-20 \mathrm{~K})$ and, less obvious also from ${ }^{13} \mathrm{CO}(7-14 \mathrm{~K})$, are higher than expected inside molecular cores if only cosmic ray ionization is considered as a heating source (a mean Galactic value of $\zeta_{\mathrm{CR}} \sim 3 \times$ $10^{-17} \mathrm{~s}^{-1}$ yields a temperature of $T \sim 8-10 \mathrm{~K}$, van der Tak \& van Dishoeck 2000). The $\mathrm{NH}_{3}$ temperatures suggest that deep inside the cores additional heating sources are present. Because the ${ }^{13} \mathrm{CO}$ is usually optically thick at the observed positions, it traces the temperature of the material at an optical depth of about unity. The actual location of this $\tau=1$ surface in the cores cannot be determined, but it is likely that the two molecules trace the temperatures at different depths in the cores.

The derived temperatures are in agreement with values found for massive and dense cores (e.g., $<20 \mathrm{~K}$ for IRDCs, Carey et al. 1998). The temperature found for $\mathrm{S} 159$ is significantly higher $(29 \mathrm{~K})$, which is consistent with its more advanced star forming state.

\subsection{Core masses and total mass}

The individual core masses $\left(M_{\mathrm{LTE}}\right)$ vary from about 20 to $1000 M_{\odot}$ (approximately $100-600 M_{\odot}$ for $M_{\text {vir }}$ ) with an average of around $190 M_{\odot}$ (about $280 M_{\odot}$ for $M_{\text {vir }}$ ). Most cores are massive when compared to low-mass dark clouds (e.g., Evans 1999, and references therein), but correspond well with masses found for high-mass protostellar objects (HMPOs; e.g., Williams et al. 2004). The LTE mass we find for S159 is in agreement with previous high resolution studies (Lebrón et al. 2001) where the core is resolved into multiple substructures.

A rough estimate for the total mass of the complex, including only the observed cores in this paper, is $3000 M_{\odot}$ (about $4500 M_{\odot}$ for $M_{\text {vir }}$ ). This is comparable to masses found for Inner Galaxy IRDCs (e.g., Carey et al. 2000).

\subsection{Physical characterization of the cores}

The observations suggest that the thermal energy is insufficient to support the cores against gravitational collapse. Additional support is seen in the observed line-widths toward the cores. These are much broader than expected from purely thermal motion (see Cols. 3-5, Table 4 and Col. 4, Table 5). The average line-width is about $1.8 \pm 0.4 \mathrm{~km} \mathrm{~s}^{-1}$. The sound speed $\Delta V_{\mathrm{s}}$, considering that the cores have a typical temperature $T$ of $15-20 \mathrm{~K}$, is given by

$\Delta V_{s}=\left(\frac{8 \ln (2) k T}{\mu m_{\mathrm{H}}}\right)^{\frac{1}{2}} \approx 0.6 \mathrm{~km} \mathrm{~s}^{-1}$,

and thus the velocities are supersonic. 


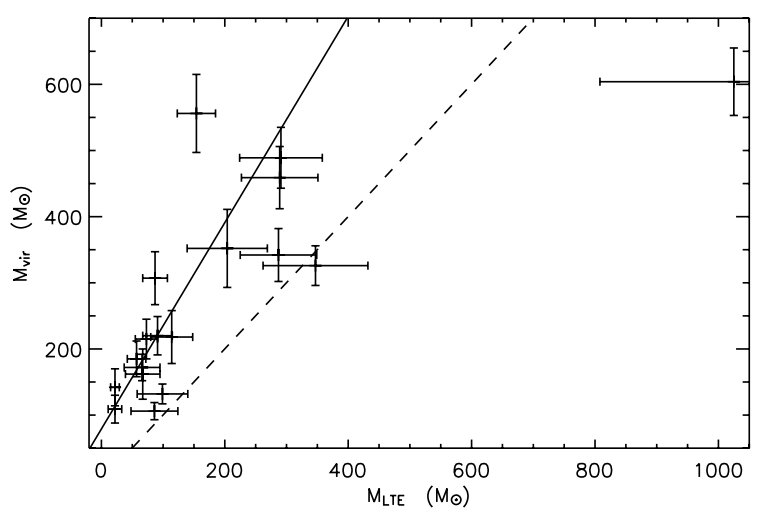

Fig. 6. The virial mass is plotted against the LTE mass. The dashed line corresponds to equal masses, where the virial parameter $\alpha=1$. The solid line is the fit to the data points.

The broad lines could be due to turbulent motions in the clouds and are indicative of regions of high-mass star formation (e.g., Myers \& Fuller 1993), in contrast to mostly thermal broadened lines often seen toward low-mass pre-stellar cores (e.g., Goodman et al. 1998). The line-width across the filament, extending over $10 \mathrm{pc}$ (at a distance of $3.1 \mathrm{kpc}$ ), is about $5 \mathrm{~km} \mathrm{~s}^{-1}$. The relation between velocity dispersion of substructures $\left(\Delta V_{l}\right)$ and the velocity dispersion over the larger complex $\left(\Delta V_{L}\right)$ in a turbulent medium can be expressed using the empirically derived Larson's law (Larson 1981);

$\Delta V_{l} \sim \Delta V_{L}\left(\frac{l}{L}\right)^{0.38}$,

where $L$ is the size of the filament and $l$ the size of the substructure. For a typical core size in our sample of $\approx 1 \mathrm{pc}$, the expected line-width is then $\approx 2 \mathrm{~km} \mathrm{~s}^{-1}$ for individual cores. The values we find are consistent with this relationship. The standard relations of mass versus line-width are, however, for much smaller cores and the larger cores presented here may be a superposition of such small cores. Extending the relation to smaller cores of $0.1 \mathrm{pc}$, the expected line-width using Eq. (14) is about 0.8 to $0.9 \mathrm{~km} \mathrm{~s}^{-1}$. This is still in agreement with values found for typical massive starless cores (e.g., Caselli \& Myers 1995).

A decay of the turbulence is expected on short timescales (see Ballesteros-Paredes et al. 2006, for a review on star formation and turbulence) and some mechanism is needed to sustain the turbulent support. Internal sources, e.g., deeply embedded YSO's may be present and stir up the material from the inside through outflows. Indicative of the presence of a heating source can be the high temperatures traced by $\mathrm{NH}_{3}$. However, we find no correlation between the line-width and the gas temperature.

The line-width could also be the result of a number of small dense clumps at slightly different velocities within the spatial resolution element of the observations. This idea is supported by the presence of multiple components along the line of sight toward many of the observed positions. In addition, the derived Jeans mass (Col. 9, Table 4) for the cores is typically $\approx 10 M_{\odot}$ and therefore, fragmentation is expected to occur on smaller mass scales compared to the observed core masses, if thermal processes dominate stability.

Figure 6 shows a plot of the virial mass versus the LTE mass. The virial parameter $\alpha$ can be defined as the ratio of the two and a value of unity corresponds to a virial equilibrium state (dashed line). The observed values for $\alpha$ range from about 1 to 3 and thus suggest that, on a global scale, the cores are close to virial equilibrium. Note that depletion of the $\mathrm{C}^{18} \mathrm{O}$ molecule may play an important role for densities in excess of $10^{4} \mathrm{~cm}^{-3}$ if $T_{\text {dust }} \sim T_{\text {gas }}$ (Bergin et al. 1995) and consequently, the LTE mass could be underestimated. Core P8 is an exceptional case where $M_{\mathrm{LTE}}>M_{\mathrm{vir}}$. As seen from the channel maps (Fig. 5, Sect. 4.2), in projection P8 appears to be a superposition of two crossing filaments. The material traced by the $\mathrm{C}^{18} \mathrm{O}$ may just be the line of sight sum of two components. Alternatively, because the components have a similar LSR velocity, a collision may be taking place between the two filaments. This would lead to the formation of a dense (perhaps gravitationally unstable) central core. If so, then we may be witnessing this scenario prior to the system being fully virialized.

\subsection{Star forming activity}

The $\mathrm{NH}_{3}$ lines are very sensitive to the temperature and therefore the presence of the higher transitions, particularly of the $(3,3)$ line, is a good indicator for the occurrence of warmer gas, possibly indicating internal heating sources. The $(3,3)$ line is clearly seen toward S159, as expected from work by Lebrón et al. (2001), and also position P5, where it may result from the presence of the associated IRAS point source. A very weak detection is seen toward positions P8 and P9.

Signatures of self-absorption in the ${ }^{13} \mathrm{CO}$ lines can be an additional indication of the presence of the warmer gas inside the cores. This may explain also the failure in fitting the ${ }^{13} \mathrm{CO}$ lines at the central velocities of the optically thin lines. Most noticeable are the positions toward S159, P5 and P8.

If embedded sources are present, they may have been identified in the 2MASS data. Figure 7 shows the $\left(H-K_{\mathrm{S}}\right)$ versus $(J-H)$ colors of the sources present in the $2 \times 2$ square degree field. The grey scaling represents the overall distribution of the field and the majority of the sources are located near the dwarf and giant sequence, indicated by the black solid curves. The spread in the colors is significant but can be addressed entirely by the use of the very faint sources in the 2MASS cata$\log$ (the faint extension) where the photometric errors are larger. The dashed lines give the reddening vectors (Rieke \& Lebofsky 1985) for the dwarf and giant sequence and the arrow corresponds to an $A_{\mathrm{V}}$ of $10 \mathrm{mag}$. In addition, the figure shows for each of the cores the associated 2MASS sources in different symbols. Note that most star colors toward the cores resemble main sequence stars with or without reddening, and these are likely reddened background or "normal" foreground stars. However, the very red $\left(H-K_{\mathrm{S}}\right)$ area right of the reddening vectors corresponds to colors of proto-stellar objects (e.g., Robitaille et al. 2006). Most promising candidates for YSO's correspond to sources associated with S159, P5 and P8.

\subsection{Notes on individual cores}

P1, P2, P3 and P7: These are regions of the molecular cloud where multiple components with enhanced densities (based on $\mathrm{C}^{34} \mathrm{~S}$ ) appear to be present along the line of sight. There are no clear indications for star forming activity from ${ }^{13} \mathrm{CO}$ self absorption, 2MASS colors or $\mathrm{NH}_{3}$ heating $\left(\mathrm{NH}_{3}\right.$ is only available for $\mathrm{P} 1$ and $\mathrm{P} 2$ ).

P4: This is part of the horizontal filamentary structure. No clear core is seen in the $\mathrm{C}^{18} \mathrm{O} 2-1$ map but high density gas is present, indicated by the $\mathrm{C}^{34} \mathrm{~S}$ line. Gravity may have induced a density enhancement along the filament. Two components are fitted by the software to the spectra, but possibly opacity effects 


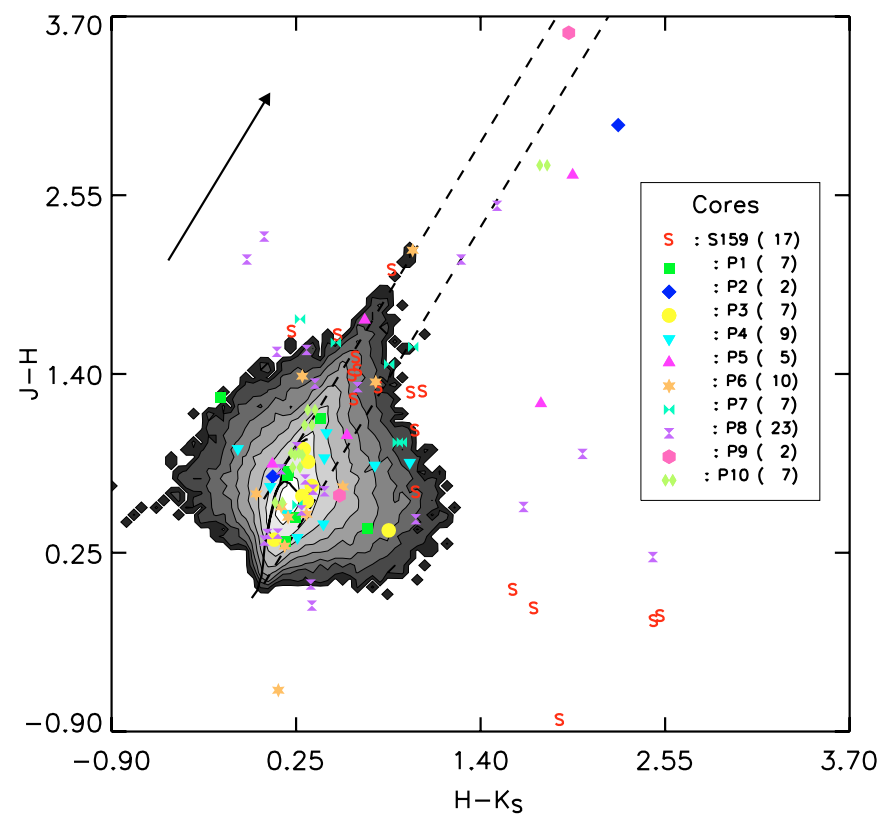

Fig. 7. 2MASS color-color plot. The grey-scales represent the location of colors of all stars in the 2 by 2 square degree field. Lighter colors correspond to a higher occurrence. The majority of the stars are situated with some spread around the dwarf and giant sequences overlaid as black curves. The arrow corresponds to a visual extinction of $10 \mathrm{mag}$, in the direction of the reddening vectors (dashed lines, Rieke \& Lebofsky 1985). The sources associated with the cores are plotted using the symbols listed in the legend. The number of associated sources is given in brackets.

cause a non-Gaussian behavior of a single component. Manual fitting of one component would result in an LTE mass of about $138 M_{\odot}$, a velocity width of $1.8 \mathrm{~km} \mathrm{~s}^{-1}$ and a virial mass of $262 M_{\odot}$.

P5: This position may be associated with, and heated by, the nearby source IRAS23137+6105, which can explain the presence of the higher $\mathrm{NH}_{3}$ transitions. One object in the 2MASS data has typical colors of a YSO. Also here, the spectra suggest that a single manual fit may be favored to two components. This would result in an LTE mass of about $220 M_{\odot}$, a velocity width of $2.57 \mathrm{~km} \mathrm{~s}^{-1}$ and a virial mass of $702 M_{\odot}$.

P6: Two dense $\left(\mathrm{C}^{34} \mathrm{~S}\right)$ components are present along the line of sight. The stellar density appears slightly enhanced but this may be a chance encounter along the line of sight. There is no indication for the presence of YSO's and none of the $\mathrm{NH}_{3}$ transitions is detected which suggests a quiescent, cold stage.

P8: This is the most profound core in the region and the best candidate for being an object similar to Inner Galaxy IRDCs (see Sect. 6.6). Automatic fitting of the CO lines resulted in two components, but in $\mathrm{C}^{34} \mathrm{~S}$, a single profile is seen. Manual fitting of a single component to the $\mathrm{CO}$ lines results in an LTE mass of about $1250 M_{\odot}$, a velocity width of $2.28 \mathrm{~km} \mathrm{~s}^{-1}$ and a virial mass of $647 M_{\odot}$. This makes the case for being an Outer Galaxy IRDC even stronger. Star formation may have started here, indicated by the $\mathrm{NH}_{3}$ lines and the presence of YSO candidates in the 2MASS color plot. Opacity effects such as self absorption due to colder foreground gas can explain the non-Gaussian appearance of the CO lines. The stellar density derived from 2MASS is significantly higher (11-13 stars per cell), compared to the rest of the field.

P9,10: There is arc-like MSX $8 \mu \mathrm{m}$ emission nearby these cores (see Figs. 1-3). This may be caused by heating due to radiation from the neighboring source IRAS 23143+6103. Such heating may also explain the detection of $\mathrm{NH}_{3}(2,2)$ and faint $\mathrm{NH}_{3}(3,3)$ emission. Compression of the material due to a shock may then lead to the observed high densities. No indication of embedded sources is seen from the 2MASS data.

\subsection{Conclusion: is $\mathrm{G111} .80+0.58$ similar to Inner Galaxy IRDCs?}

The global characteristics of the G111.80+0.58 complex that are presented in this paper, such as its size $(\sim 10 \mathrm{pc})$, column density (peak $\left.>10^{22} \mathrm{~cm}^{-2}\right)$ and LTE mass $\left(\sim 3000 M_{\odot}\right)$ indicate a clear resemblance to the bulk properties of Inner Galaxy IRDCs (Simon et al. 2006). However, except for P8, the individual core properties (e.g., size: $\sim 1$ pc, LTE mass: $20-350 M_{\odot}$, density: $10^{3}-10^{4} \mathrm{~cm}^{-3}$ ) fall short of the values found for compact (sub)mm cores in IRDCs (size: $0.02-0.8 \mathrm{pc}$, mass: $10-10^{3} M_{\odot}$, density: $10^{3}-10^{7} \mathrm{~cm}^{-3}$ Rathborne et al. 2006).

Would this cloud be seen in extinction were it observed toward a bright mid-IR background?

A typical column density found for the cores is $10-20 \times$ $10^{21} \mathrm{~cm}^{-2}$, which corresponds to an $A_{\mathrm{V}}$ of 5-10 mag (Bohlin et al. 1978). The corresponding extinction at $8 \mu \mathrm{m}$ can be expressed as $A_{8 \mu \mathrm{m}} \sim 0.04 A_{\mathrm{V}}<0.4$ mag, using conversions adopted from Indebetouw et al. (2005) and Rieke \& Lebofsky (1985). Typical extinctions around $8 \mu \mathrm{m}$ for Inner Galaxy IRDCs are 1-2 mag (Carey et al. 2000). Thus, based on this simple reasoning the cloud would not meet the IRDC criterion of mid-IR extinction. However, following the same analysis, the extinction toward position $\mathrm{P} 8\left(N \sim 74 \times 10^{21} \mathrm{~cm}^{-2}\right)$ is about 1.4 mag at $8 \mu \mathrm{m}$. Considering that the above values represent the densest component of the two $\mathrm{CO}$ fits toward $\mathrm{P} 8$, the actual $8 \mu \mathrm{m}$ extinction is even higher when using a single fit (1.8 mag). This central part of the cloud makes the region a very promising Outer Galaxy IRDC candidate. The presence of possible embedded heating sources at this position may indicate that the core is not in a very early, quiescent state, but already forming stars. Recent studies (Rathborne et al. 2005; Ormel et al. 2005, v.d. Wiel, privat comm.), using submm and Spitzer data indicate that some Inner Galaxy IRDCs are presently forming stars in their cores and along the filaments as well.

Obviously, the dark cloud candidate presented in this paper is in the vicinity of star forming activity (NGC 7538, S159) and in that sense the boundary conditions may not be that deviant from Inner Galaxy IRDCs. It should be noted however, that there are environmental differences in the Outer Galaxy (e.g., radiation field, density, abundance; Brand \& Wouterloot 1995; Rudolph et al. 2006, and references therein) when compared to the inner spiral arms and the Molecular Ring, where most of the IRDCs are found.

The super-thermal line-widths and the presence of massive, cold and dense cores in G111.80+0.58 are both indicative of a high-mass star forming complex. The combined mass of the cores is perhaps too low to form a massive star cluster (like Orion). However, this region is part of a much larger molecular cloud complex. We conclude that the G111.80+0.58 complex likely belongs to a category of objects similar to intermediatemass IRDCs (e.g., IRDC G48 toward W51; Ormel et al. 2005) in an early, but not pristine stage of star formation.

\section{Concluding remarks}

Radiative transfer codes can provide a better understanding of the properties of the gas, e.g., radial density and temperature profiles can be investigated, but require more detailed observations. 
We did conduct tests with a multi-zone escape probability code $(\beta 3 \mathrm{D}$, Poelman \& Spaans 2006, 2005) and these resulted in similar temperature, density and mass estimates as presented in this paper. We therefore conclude that for the integrated properties these codes have no additional contribution to the results. The main advantage when using these models is getting a better understanding of the line profiles. In particular, effects such as self absorption or velocity structures, like infall motion may explain some of the double peaked and non-Gaussian features (see e.g., Evans 1999). In the work presented here, non-Gaussian features are treated as separate components. Note in this respect that different optically thin lines (e.g., $\mathrm{C}^{18} \mathrm{O}$ and $\mathrm{C}^{34} \mathrm{~S}$ for core $\mathrm{P} 1$ ) show their peak emission at opposite sides of the central velocity $V_{\mathrm{LSR}}$.

High spatial and spectral resolution in addition with radiative transfer models are required in the future to get better constraints on the physical properties on smaller scales. A spatial resolution at sub-arcsecond scales may resolve the presence of small high density clumps. Resolving this substructure will allow an investigation of the Dense Clump Mass Function (DCMF). It is of great importance to conduct these studies to put constraints on the origin of the stellar mass spectrum. Not only in nearby, generally low mass star forming clouds (e.g., Pipe Nebula; Lombardi et al. 2006), but particularly in the more massive and distant IRDCs, including the Outer Galaxy region presented in this paper.

A different way of characterizing IRDCs is by using (sub)mm continuum observations. All Inner Galaxy IRDCs show strong submm emission (e.g., Carey et al. 2000) and some contain bright, centrally peaked cores. In addition, these data allow an independent measurement of properties such as temperature, mass and luminosity. It will be worthwhile to observe the G111.80+0.58 cloud in dust continuum and compare the data with the molecular line results presented here and with existing submm studies in the Inner Galaxy.

Acknowledgements. We thank the anonymous referee for his/her careful reading of the manuscript and his/her constructive remarks.

\section{References}

Bachiller, R., Guilloteau, S., \& Kahane, C. 1987, A\&A, 173, 324

Ballesteros-Paredes, J., Klessen, R. S., Mac Low, M., \& Vazquez-Semadeni, E. 2006, ArXiv Astrophysics e-prints

Bergin, E. A., \& Langer, W. D. 1997, ApJ, 486, 316

Bergin, E. A., Langer, W. D., \& Goldsmith, P. F. 1995, ApJ, 441, 222

Blitz, L., Fich, M., \& Stark, A. A. 1982, ApJS, 49, 183

Bohlin, R. C., Savage, B. D., \& Drake, J. F. 1978, ApJ, 224, 132

Bourke, T. L., Garay, G., Lehtinen, K. K., et al. 1997, ApJ, 476, 781

Brand, J., \& Blitz, L. 1993, A\&A, 275, 67

Brand, J., \& Wouterloot, J. G. A. 1995, A\&A, 303, 851
Brunt, C. M., Kerton, C. R., \& Pomerleau, C. 2003, ApJS, 144, 47 Carey, S. J., Clark, F. O., Egan, M. P., et al. 1998, ApJ, 508, 721

Carey, S. J., Feldman, P. A., Redman, R. O., et al. 2000, ApJ, 543, L157

Caselli, P., \& Myers, P. C. 1995, ApJ, 446, 665

Chini, R., \& Wink, J. E. 1984, A\&A, 139, L5

Dickman, R. L. 1978, ApJS, 37, 407

Egan, M. P., Shipman, R. F., Price, S. D., et al. 1998, ApJ, 494, L199

Evans, N. J. 1999, ARA\&A, 37, 311

Forveille, T., Guilloteau, S., \& Lucas, R. 1989, Grenoble: IRAM

Frerking, M. A., Langer, W. D., \& Wilson, R. W. 1982, ApJ, 262, 590

Frieswijk, W. W. F., \& Shipman, R. F. 2007, in preperation

Garay, G., Faúndez, S., Mardones, D., et al. 2004, ApJ, 610, 313

Goodman, A. A., Barranco, J. A., Wilner, D. J., \& Heyer, M. H. 1998, ApJ, 504, 223

Heyer, M. H., Brunt, C., Snell, R. L., et al. 1998, ApJS, 115, 241

Indebetouw, R., Mathis, J. S., Babler, B. L., et al. 2005, ApJ, 619, 931

Johnstone, D., Fiege, J. D., Redman, R. O., Feldman, P. A., \& Carey, S. J. 2003, ApJ, 588, L37

Kameya, O., Morita, K.-I., Kawabe, R., \& Ishiguro, M. 1990, ApJ, 355, 562

Klessen, R. S., Ballesteros-Paredes, J., Li, Y., \& Mac Low, M.-M. 2004, in The Formation and Evolution of Massive Young Star Clusters, ed. H. J. G. L. M. Lamers, L. J. Smith, \& A. Nota, ASP Conf. Ser., 322, 299

Lada, C. J., Lada, E. A., Clemens, D. P., \& Bally, J. 1994, ApJ, 429, 694

Larson, R. B. 1981, MNRAS, 194, 809

Larson, R. B. 1985, MNRAS, 214, 379

Lebrón, M. E., Rodríguez, L. F., \& Lizano, S. 2001, ApJ, 560, 806

Lombardi, M., Alves, J., \& Lada, C. J. 2006, A\&A, 454, 781

MacLaren, I., Richardson, K. M., \& Wolfendale, A. W. 1988, ApJ, 333, 821

Menten, K. M., Pillai, T., \& Wyrowski, F. 2005, in IAU Symp., ed. R. Cesaroni, M. Felli, E. Churchwell, \& M. Walmsley, 23

Myers, P. C., \& Fuller, G. A. 1993, ApJ, 402, 635

Ormel, C. W., Shipman, R. F., Ossenkopf, V., \& Helmich, F. P. 2005, A\&A, 439, 613

Ott, M., Witzel, A., Quirrenbach, A., et al. 1994, A\&A, 284, 331

Pérault, M., Omont, A., Simon, G., et al. 1996, A\&A, 315, L165

Poelman, D. R., \& Spaans, M. 2005, A\&A, 440, 559

Poelman, D. R. \& Spaans, M. 2006, A\&A, 453, 615

Rathborne, J. M., Jackson, J. M., Chambers, E. T., et al. 2005, ApJ, 630, L181

Rathborne, J. M., Jackson, J. M., \& Simon, R. 2006, ApJ, 641, 389

Rieke, G. H., \& Lebofsky, M. J. 1985, ApJ, 288, 618

Robitaille, T. P., Whitney, B. A., Indebetouw, R., Wood, K., \& Denzmore, P. 2006, ApJS, 167, 256

Rudolph, A. L., Fich, M., Bell, G. R., et al. 2006, ApJS, 162, 346

Sandell, G., \& Sievers, A. 2004, ApJ, 600, 269

Schuster, K.-F., Boucher, C., Brunswig, W., et al. 2004, A\&A, 423, 1171

Shipman, R. F., Frieswijk, W., \& Helmich, F. P. 2003, in Galactic Star Formation Across the Stellar Mass Spectrum, ed. J. M. De Buizer, \& N. S. van der Bliek, ASP Conf. Ser., 287, 252

Simon, R., Rathborne, J. M., Shah, R. Y., Jackson, J. M., \& Chambers, E. T. 2006, ApJ, 653, 1325

Skrutskie, M. F., Cutri, R. M., Stiening, R., et al. 2006, AJ, 131, 1163

Tafalla, M., Myers, P. C., Caselli, P., \& Walmsley, C. M. 2004, A\&A, 416, 191

van der Tak, F. F. S., \& van Dishoeck, E. F. 2000, A\&A, 358, L79

van der Tak, F. F. S., van Dishoeck, E. F., Evans, II, N. J., \& Blake, G. A. 2000, ApJ, 537, 283

Werner, M. W., Becklin, E. E., Gatley, I., et al. 1979, MNRAS, 188, 463

Williams, S. J., Fuller, G. A., \& Sridharan, T. K. 2004, VizieR Online Data Catalog, 341, 70115 
W. W. F. Frieswijk et al.: Physical characteristics of an Outer Galaxy dark cloud, Online Material p 1

\section{Online Material}




\section{Appendix A: Calibration of data obtained at the 100-m telescope}

At the frequency considered here $(23 \mathrm{GHz})$, the Effelsberg telescope offers a calibration based on noise diodes, so that the received signal is not converted into $\mathrm{K}$ but into internal receiver counts. In order to both convert the receiver counts into antenna temperature, as well as to correct for the atmospherical absorption, we have observed a photometric calibrator (here NGC 7027) at various elevations in the course of each observing night. This approach is similar to the so-called "antenna tipping" used to measure the sky opacity during e.g., bolometer observations. The weather conditions were stable enough to assume that the opacity derived through this technique was reasonably representative of the daily set of data. If $F_{\text {int }}$ is the conversion factor between internal counts and Jy above the atmosphere and corrected for backward losses, the measured receiver counts are (in a given spectrometer channel):

$T_{\mathrm{A}}^{\mathrm{int}}=G(\mathrm{el}) \times \mathrm{e}^{\left(-\tau_{\mathrm{z}} \mathrm{A}\right)}, \times \frac{\mathrm{T}_{\mathrm{A}}^{*} \eta_{\ell}}{\mathrm{F}_{\mathrm{int}}}$

where $A$ is the airmass at elevation el, $\tau_{z}$ the zenith opacity and $G(\mathrm{el})$ the normalized elevation gain. Observing a photometric calibrator of known flux $S_{v, \text { ref }}$ one has:

$T_{\mathrm{A}, \mathrm{ref}}^{*}=\frac{A_{\mathrm{geom}} \eta_{A}}{2 k \eta_{\ell}} S_{\nu, \text { ref }}=\frac{G_{(\mathrm{K} / \mathrm{Jy})}}{\eta_{\ell}} S_{\nu, \text { ref }}$

and

$\log \left(\frac{G_{(\mathrm{K} / \mathrm{Jy})} S_{v, \text { ref }}}{T_{\mathrm{A}}^{\text {int }}}\right)=\log \left(\frac{F_{\text {int }}}{G(\mathrm{el})}\right)+\frac{\tau_{z}}{\sin (\mathrm{el})}$.

Using the calibrated flux published by Ott et al. (1994) and observing the reference at various elevations, the calibration procedure thus consists of fitting the previous expression with a pair of $\left(F_{\text {int }}, \tau\right)$, assuming that the opacity has not significantly changed in the course of the observations. The antenna temperature is then given by:

$T_{\mathrm{A}}^{*}=\frac{T_{\mathrm{A}}^{\mathrm{int}} \times G_{(\mathrm{K} / \mathrm{Jy})} \times F_{\text {int }}}{G(\mathrm{el}) \times \mathrm{e}^{\left(-\tau_{\mathrm{z}} \mathrm{A}\right)} \times \eta_{\ell}}$.

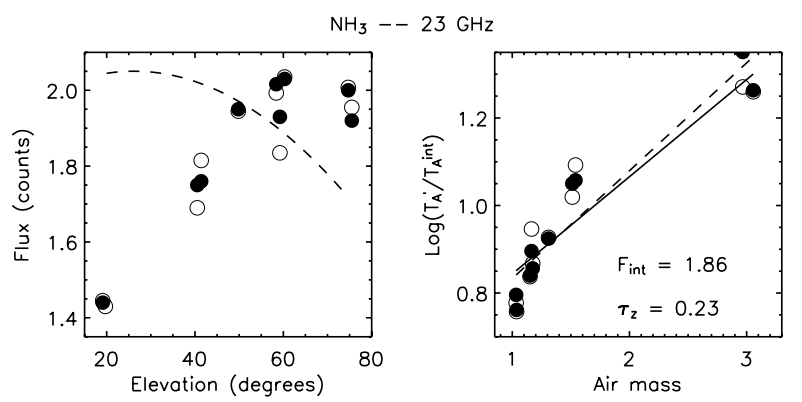

Fig. A.1. Example of Effelsberg calibration measurements taken on NGC 7027 for one day at the two frequencies considered here. Left: flux measurements for azimuthal (black circles) and horizontal (white circles) scans. The elevation gain curve is plotted, normalized to the highest flux of the graph. Right: fit of Eq. (A.2) on data collected during a single day for azimuthal (full line) and horizontal (dashed lines) data. The resulting $\tau_{z}$ and $F_{\text {int }}$ are the mean of these two fits.

The NGC 7027 flux at $23 \mathrm{GHz}$ was taken as 5.11 Jy. We derived a conversion gain of $1.86 \pm 0.1$ and the opacity was around 0.2 , consistent with expected atmospherical transmission in the centrimetric domain. Figure A.1 illustrates the steps involved in the calibration process. It shows the combined effect of antenna gain and opacity for data taken at various elevations. Finally, assuming that our sources emit in a solid angle comparable to the main beam, the derived fluxes are translated into antenna main beam temperatures using the antenna efficiencies provided by the 100-m staff (A. Kraus, priv. comm.).

\section{Appendix B: Figures}


W. W. F. Frieswijk et al.: Physical characteristics of an Outer Galaxy dark cloud, Online Material p 3
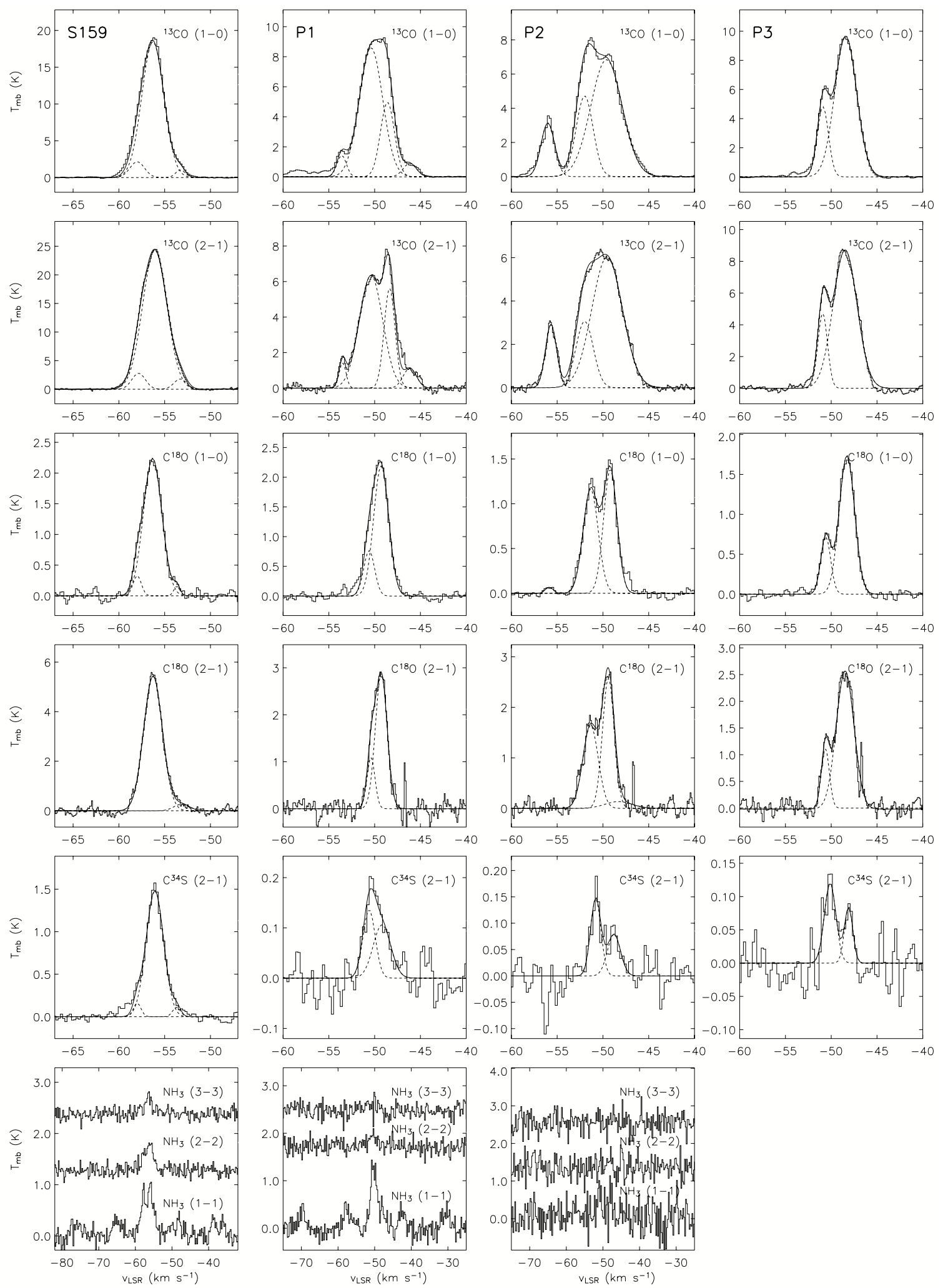

Fig. B.1. Observed spectra (solid lines) and for the IRAM data the Gaussian fits (dashed lines) for the positions listed in Table 1. Each column contains the spectra of the position labeled at the top in the following order: ${ }^{13} \mathrm{CO} 1-0,{ }^{13} \mathrm{CO} 2-1, \mathrm{C}^{18} \mathrm{O} 1-0, \mathrm{C}^{18} \mathrm{O} 2-1, \mathrm{C}^{34} \mathrm{~S} 2-1$ and $\mathrm{NH}_{3}$. Missing panels were not observed. 
W. W. F. Frieswijk et al.: Physical characteristics of an Outer Galaxy dark cloud, Online Material p 4
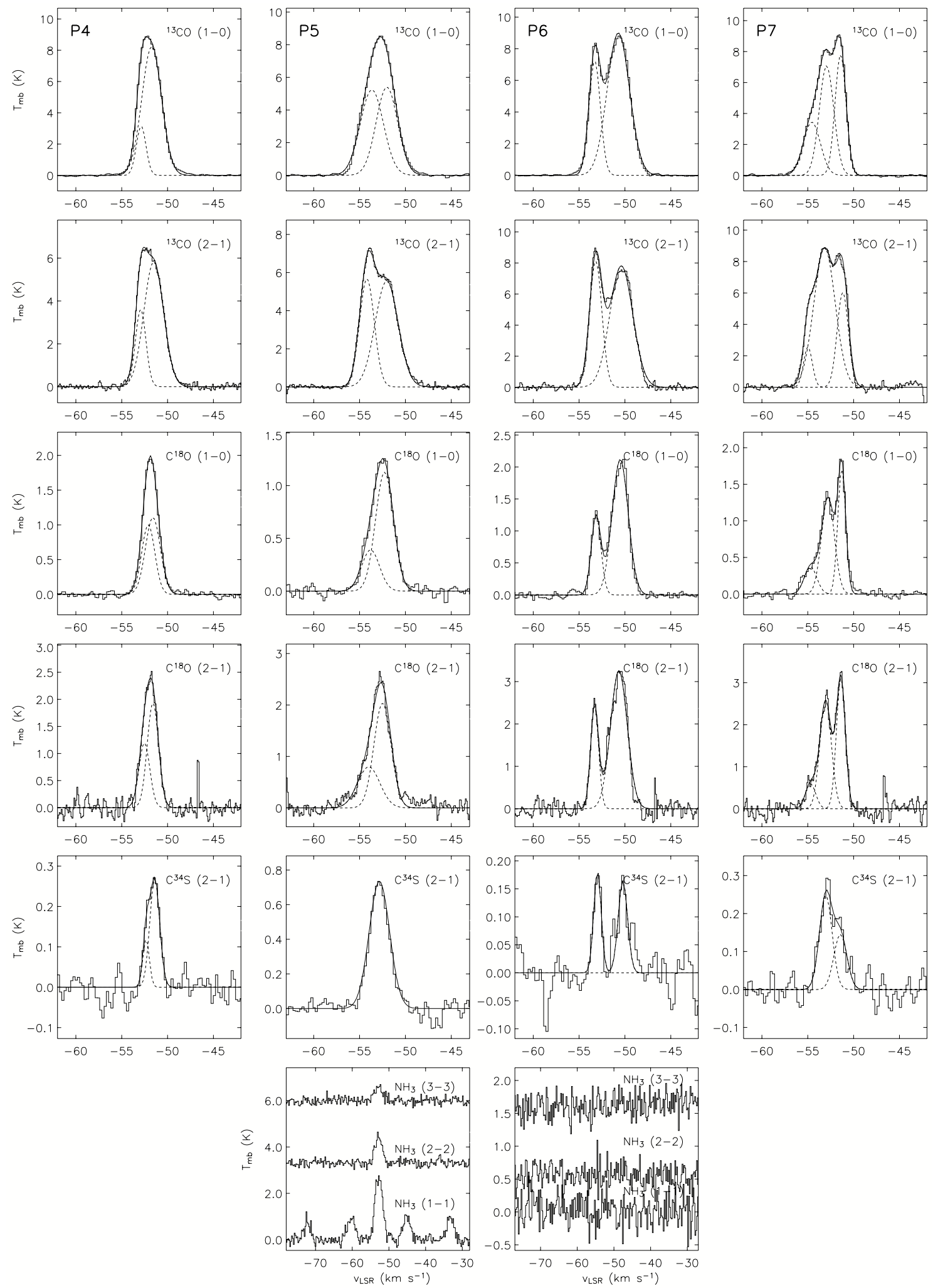

Fig. B.2. Same as Fig. B.1. 
W. W. F. Frieswijk et al.: Physical characteristics of an Outer Galaxy dark cloud, Online Material p 5
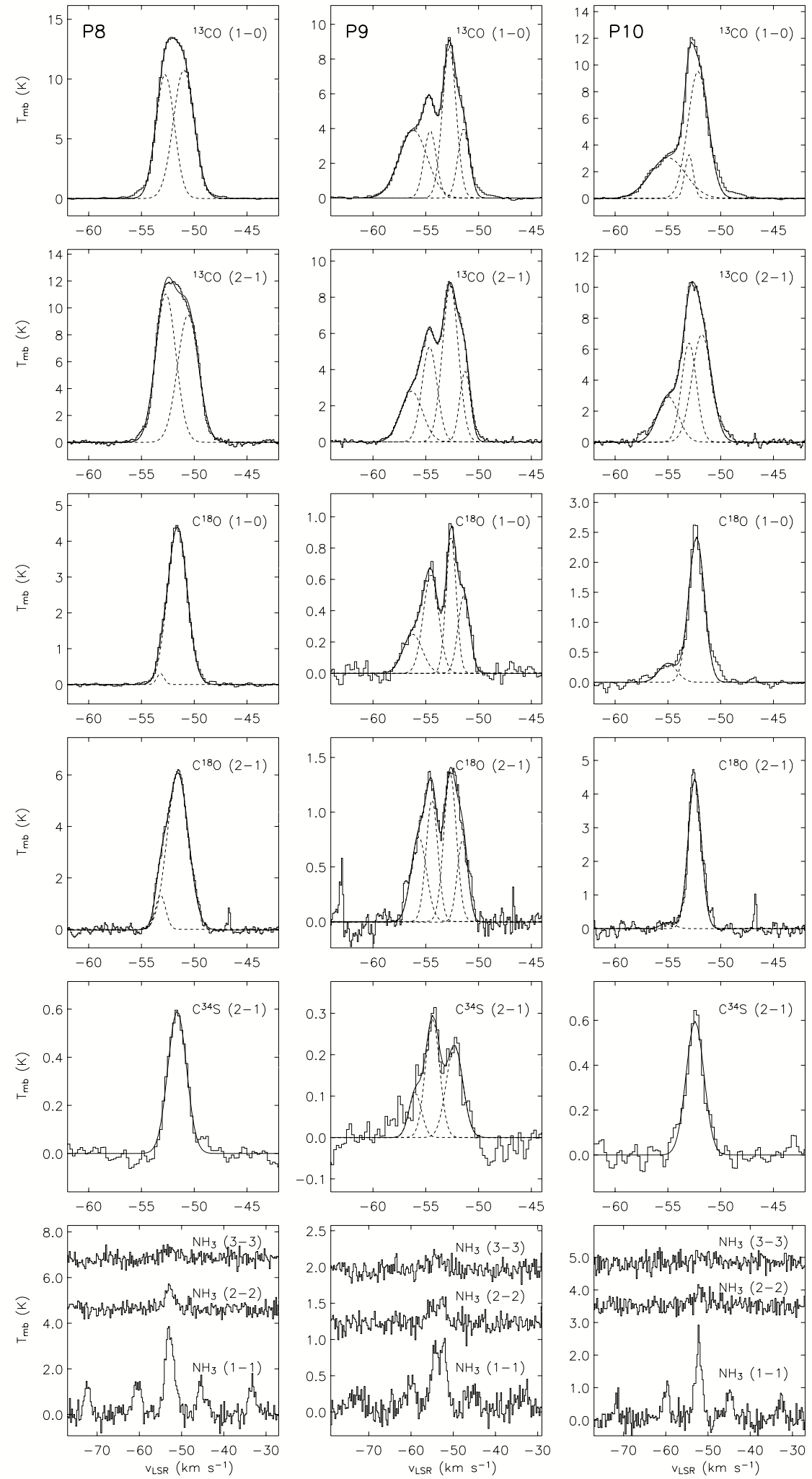

Fig. B.3. Same as Fig. B.1. 